\title{
Loops de Bol algébricos e analíticos
}

\author{
Márcio Alexandre de Oliveira Reis
}

\author{
TESE APRESENTADA \\ $\mathrm{AO}$ \\ Instituto DE MATEMÁTICA E EstatísticA \\ DA \\ UniversidAdE DE SÃo PAUlo \\ PARA \\ OBTENÇÃO DO TÍTULO \\ $\mathrm{DE}$ \\ DOUTOR EM CIÊNCIAS
}

\author{
Programa: Matemática \\ Orientador: Prof. Dr. Alexandre Grichkov
}

Durante o desenvolvimento deste trabalho o autor recebeu auxílio financeiro do CAPES

São Paulo, maio de 2010 


\title{
Loops de Bol algébricos e analíticos
}

\author{
Este exemplar corresponde à redação \\ final da tese devidamente corrigida \\ e defendida por Márcio Alexandre de Oliveira Reis \\ e aprovada pela Comissão Julgadora.
}

Banca Examinadora:

- Prof. Dr. Alexandre Grichkov (Presidente) - IME-USP.

- Prof. Dr. Guilherme Augusto de La Rocque Leal - UFRJ.

- Prof. Dr. Alexandr Zubkov - OMSK STATE.

- Prof. Dr. Juan Carlos Gutiérrez Fernández - IME-USP.

- Prof. Dr. Maria de Lourdes Merlini Giuliani - UFABC. 


\section{Agradecimentos}

A Deus, pela minha vida, por tudo que tenho e por todas as oportunidades que me foram dadas.

Aos meus queridos pais, Zilda e Florêncio, à minha esposa Cristina por estar sempre ao meu lado, por seu amor e apoio e por nunca ter deixado eu desistir.

Ao professor Alexandre Grichkov pela orientação..

Ao Instituto de Matemática e Estatística pela oportunidade de aprendizagem e a disponibilização de seus recursos para meus estudos.

A Capes pelo auxílio financeiro indispensável à realização desta tese. 


\section{Resumo}

Neste trabalho classificamos, a menos de isomorfismos, as álgebras de Bol de dimensão 2 sobre um corpo de característica 0. Também determinamos suas álgebras de Lie envolvente e, mostramos que existem álgebras de Bol não isomorfas cujas álgebras de Lie envolventes coorrespondentes são isomorfas. Calculamos os grupos algébricos (locais) correspondentes a cada uma das álgebras de Lie envolventes e provamos que todo loop de Bol analítico (algébrico) global de dimensão 2 sobre um corpo de característica 0 é um grupo. Exibimos exemplos de loops de Bol algébricos globais de dimensão $n$, para todo $n>2$, e fornecemos uma condição necessária e suficiente para a existência de um loop de Bol algébrico global quando a álgebra de Bol tem uma álgebra de Lie envolvente nilpotente de índice 2 sobre um corpo de característica diferente de 2 .

Palavras-chave: Álgebras de Lie, grupos algébricos, álgebras de Bol, álgebra de Lie envolvente, loops de Bol, loops de Bol algébricos, loops de Bol analíticos. 


\section{Abstract}

In this work, we classify up to isomorphism, the Bol algebras of dimension 2 over a field of characteristic 0 . We also determine their enveloping Lie algebras and we exhibit two non-isomorphic Bol algebras which have isomorphic enveloping Lie algebras. We determine the (local) correspondent algebraic groups of each of those enveloping Lie algebras and we show that every global analytic (algebraic) Bol loop of dimension 2 over a field of characteristic 0 is a group. We exhibit examples of non-nilpotent solvable algebraic Bol loops in dimension $n$ for every $n>2$, and we were able to give a necessary and sufficient condition to decide if a local algebraic Bol loop is global when its enveloping Lie algebra is nilpotent of index 2 and $\operatorname{char}(\mathbb{F}) \neq 2$.

Keywords: Lie algebras, algebraic groups, Bol algebras, enveloping Lie algebras, Bol loops, algebraic Bol loops, analytic Bol loops. 


\section{Sumário}

Capítulo 1: Preliminares $\quad 4$

1.1 Álgebras de Lie . . . . . . . . . . . . . . . . . . . . . . . 4

1.2 Grupos Algébricos . . . . . . . . . . . . . . . 6

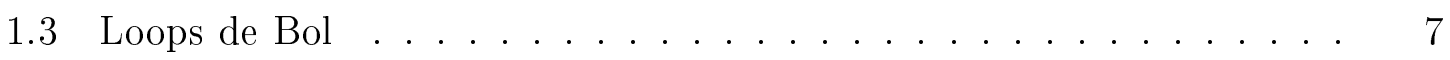

1.4 Álgebras de Bol . . . . . . . . . . . . . . . . . . . . . . . . . . 9 9

1.5 Álgebra tangente de um loop de Bol . . . . . . . . . . . . . . 17

Capítulo 2: Álgebras de Bol de dimensão $2 \quad 19$

2.1 Álgebras de Bol de dimensão 2 sobre o corpo algebricamente fechado 19

2.2 Álgebra Envolvente Minimal . . . . . . . . . . . . . . . . . . . . 31

2.3 Álgebras de Bol de dimensão 2 sobre um corpo que não é algebricamente fechado . . . . . . . . . . . . . . . . 33

Capítulo 3: Loops de Bol algébricos e analíticos de dimensão $2 \quad 36$

3.1 Loops de Bol algébricos e analíticos de dimensão 2 sobre um corpo algebricamente fechado . . . . . . . . . . . . . . 36

3.2 Loops de Bol algébricos de dimensão 2 sobre corpos que não são algebricamente fechados . . . . . . . . . . . . . . 44

Capítulo 4: Loops de Bol Algébricos Globais e Álgebras de Bol Críticas 58

4.1 Loops de Bol Algébricos . . . . . . . . . . . . . . . . . 58

4.2 Álgebras de Bol Críticas . . . . . . . . . . . . . . . . 62

Capítulo 5: Loops de Bol unipotentes de grau de nilpotência dois $\quad 65$ 
5.1 Loops de Bol unipotentes de grau de nilpotência dois . . . . . . . . .

Referências Bibliográficas 


\section{Introdução}

Lembremos que um loop é um conjunto $L$ munido com uma operação binária $\cdot$ tal que 1 é o elemento identidade e as equações

$$
a \cdot x=b \text { e } a \cdot y=b
$$

têm soluções únicas em $L$. Nesta tese, estudaremos aqueles loops que satisfazem a identidade de Bol à direita, isto é,

$$
(x y \cdot z) y=x(y z \cdot y)
$$

para quaisquer $x, y, z \in L$. Esta classe de loops foi introduzida por Gerrit Bol, em 1937, num contexto geométrico.

Um espaço vetorial $\mathcal{B}$, sobre um corpo $\mathbb{K}$, é chamado uma álgebra de Bol se ela tem uma operação binária $(\cdot, \cdot)$ e uma operação trilinear $(\cdot ; \cdot, \cdot)$ que são lineares em todas as variáveis e valem as seguintes identidades,

$$
\begin{gathered}
(x ; y, y)=0 \\
(x ; y, z)+(y ; z, x)+(z ; x, y)=0 \\
((x ; y, z) ; u, v)=((x ; u, v) ; y, z)+(x ;(y ; u, v), z)+(x ; y,(z ; u, v)) \\
x \cdot x=0 \\
(x \cdot y ; u, v)=(x ; u, v) \cdot y+x \cdot(y ; u, v)+(u \cdot v ; x, y)+(x \cdot y) \cdot(u \cdot v)
\end{gathered}
$$

para quaisquer $x, y, z, u, v \in \mathcal{B}$.

As álgebras de Bol foram introduzidas em [12] no contexto de um estudo de loops de Bol, definidos pela identidade de Bol à direita. Estas álgebras desempenham o 
mesmo papel com relação aos loops de Bol que as álgebras de Lie com relação aos grupos de Lie ou as álgebras de Mal'tsev com relação aos loops de Moufang (Veja também [13]).

Esta tese está dividida nos seguintes capítulos.

No capítulo 1 são definidos os conceitos com os quais trabalharemos durante o desenvolvimento dela. Nem todos foram definidos para evitar se estender demasiadamente, mas referências bibliográficas foram dadas para facilitar a localização destes.

No Capítulo 2, estudamos as álgebras de Bol de dimensão 2 sobre corpos de característica 0 . Como nós sabemos, este estudo foi feito por [6], mas não foi publicado em revistas de grande circulação. Por isso neste capítulo, classificamos completamente estas álgebras e damos critérios para decidir quando duas delas são isomorfas. Ressaltamos também que enquanto existem apenas duas álgebras de Lie de dimensão 2 não isomorfas, a situação é bem diferente no caso de álgebras de Bol de dimensão 2. Também exibimos suas álgebras de Lie envolventes e mostramos que existem álgebras de Bol não isomorfas cujas álgebras de Lie envolventes correspondentes são isomorfas.

No Capítulo 3, depois de calcular os grupos algébricos correspondentes às álgebras de Lie envolventes de cada uma das álgebras de Bol obtidas no Capítulo 2, provamos que cada loop de Bol analítico ou algébrico global de dimensão 2 sobre um corpo de característica 0 é um grupo.

O Capítulo 4 foi motivado pela seguinte conjectura (G.Nagy): Seja $B$ um loop de Bol algébrico ou analítico sobre um corpo algebricamente fechado de característica 0 . Se $\operatorname{Mult}(B)$ é nilpotente, então $B$ é nilpotente.

Na seção 1 do Capítulo 4 exibimos um contra-exemplo $B$ a esta conjectura. Destacamos que qualquer subálgebra ou quociente da álgebra de Bol $\mathcal{B}$ correspondente é é nilpotente, mas $B$ não é nilpotente. Vamos chamar as álgebras de Bol com estas propriedades de críticas. No final deste capítulo construímos álgebras de Bol críticas de dimensão arbitrária.

Finalmente, no capítulo 5, provamos o principal resultado desta tese: Seja $\mathcal{B}$ uma álgebra de Bol cuja álgebra de Lie envolvente é nilpotente de índice de nilpotência 2. Então o loop de Bol $B$ correspondente é global se, e somente se, $\mathcal{B}$ é de Engel. Vamos lembrar que uma álgebra é Engel se existe $n$ tal que $R_{x}^{n}=0$ para todos $x \in B$. 
Este resultado justifica a seguinte Conjectura: Seja $\mathcal{B}$ uma álgebra de Bol sobre um corpo de característica 0 tal que $\operatorname{Lie}(\mathcal{B})$ é nilpotente. Então o loop de Bol algébrico correspondente é global se, e somente se, $\mathcal{B}$ é de Engel. 


\section{Capítulo 1}

\section{Preliminares}

Neste capítulo iremos dar as definições e enunciar os resultados que serão utilizados ao longo desta tese. Em todo este trabalho, $\mathbb{K}$ denotará um corpo de característica 0 salvo menção contrária.

\section{$1.1 \quad$ Álgebras de Lie}

As definições e resultados desta seção foram tiradas de [8] e [15].

Definição 1.1.1: Uma álgebra de Lie consiste de um espaço vetorial $\mathfrak{g}$ sobre o corpo $\mathbb{K}$, munido de um produto $[\cdot, \cdot]: \mathfrak{g} \rightarrow \mathfrak{g}$ com as seguintes propriedades:

1) $[\cdot, \cdot]$ é bilinear,

2) $[x, x]=0$ para todo $x \in \mathfrak{g}$,

3) satisfaz a identidade de Jacobi, isto é,

$$
[x,[y, z]]+[y,[z, x]]+[z,[x, y]]=0
$$

para quaisquer $x, y, z \in \mathfrak{g}$.

Seja $\mathfrak{g}$ uma álgebra de Lie. Um subespaço $\mathfrak{h} \subset \mathfrak{g}$ é uma subálgebra de Lie (resp. um ideal) se $[x, y] \in \mathfrak{h}$ para quaisquer $x, y \in \mathfrak{h}($ resp. $[x, \mathfrak{h}] \in \mathfrak{h}$, para todo $x \in \mathfrak{g})$.

A seguir daremos alguns exemplos de álgebras de Lie e fixaremos algumas notações. 
Exemplo 1.1.2: 1) Seja $A$ uma $\mathbb{K}$-álgebra associativa. Então $A$ tem uma estrutura de álgebra de Lie dada por: para quaisquer $x, y \in A$, definimos $[x, y]=x y-y x$. Por exemplo, $\mathfrak{g l}(n, \mathbb{K})$ denota a álgebra de Lie das matrizes $n \times n$ sobre o corpo $\mathbb{K}$, com o produto definido anteriormente.

2) $\mathfrak{s l}(n, \mathbb{K})$ denota o conjunto das matrizes em $\mathfrak{g l}(n, \mathbb{K})$ cujo traço é zero.

3) $\mathfrak{t}(n, \mathbb{K})$ denota o conjunto das matrizes triangulares superiores em $\mathfrak{g l}(n, \mathbb{K})$.

4) $\mathfrak{d}(n, \mathbb{K})$ denota o conjunto das matrizes diagonais em $\mathfrak{g l}(n, \mathbb{K})$.

Definição 1.1.3: Sejam $\mathfrak{g}$ e $\mathfrak{h}$ duas álgebras de Lie. Uma transformação linear $T: \mathfrak{g} \rightarrow \mathfrak{h}$ é:

1) um homomorfismo de álgebras de Lie se $T[x, y]=[T x, T y]$, para quaisquer $x, y \in \mathfrak{g}$.

2) um isomorfismo de álgebras de Lie se for um homomorfismo inversível.

3) um automorfismo de álgebras de Lie se é um isomorfismo e $\mathfrak{g}=\mathfrak{h}$.

4) uma automorfismo involutivo se $T^{2}=\mathrm{Id}$.

Como um exemplo de homomorfismo de álgebras de Lie temos: para cada elemento $x \in \mathfrak{g}$, onde $\mathfrak{g}$ é uma álgebra de Lie, a aplicação $a d(x): \mathfrak{g} \rightarrow \mathfrak{g}$ definida por $\operatorname{ad}(x)(y)=$ $[x, y]$ é uma transformação linear, e aplicação ad: $\mathfrak{g} \rightarrow \mathfrak{g l}(n, \mathbb{K})$ definida por ad : $x \mapsto a d(x)$ é um homomorfismo de álgebras de Lie. O homomorfismo ad é chamado representação adjunta.

Sejam $A$ e $B$ dois subconjuntos de uma álgebra de Lie g. A notação $[A, B]$ indicará o subespaço de $\mathfrak{g}$ gerado por

$$
\{[a, b] \mid a \in A, b \in B\} .
$$

Define-se, por indução, os seguintes subespaços de uma álgebra de Lie $\mathfrak{g}$ :

$$
\mathfrak{g}^{(0)}=\mathfrak{g}, \mathfrak{g}^{\prime}=[\mathfrak{g}, \mathfrak{g}], \ldots, \mathfrak{g}^{(i+1)}=\left[\mathfrak{g}^{(i)}, \mathfrak{g}^{(i)}\right], \ldots
$$

Cada um desses espaços é ideal de $\mathfrak{g}$ e, além disso, $\mathfrak{g}^{(i+1)} \subset \mathfrak{g}^{(i)}[15]$.

Definição 1.1.4: Uma álgebra de Lie $\mathfrak{g}$ é solúvel se $\mathfrak{g}^{(k)}=\{0\}$ para algum $k \geq 1$. 
Também por indução, definimos os seguintes subespaços de $\mathfrak{g}$ :

$$
\mathfrak{g}^{1}=\mathfrak{g}, \quad \mathfrak{g}^{2}=[\mathfrak{g}, \mathfrak{g}], \quad \mathfrak{g}^{k}=\left[\mathfrak{g}, \mathfrak{g}^{k-1}\right], \ldots
$$

Como antes, prova-se que $\mathfrak{g}^{k}$ é um ideal de $\mathfrak{g}$ para todo $k \geq 1$ e, além disso $\mathfrak{g}^{k+1} \subset \mathfrak{g}^{k}$.

Definição 1.1.5: Uma álgebra de Lie $\mathfrak{g}$ é nilpotente se $\mathfrak{g}^{k}=\{0\}$ para algum $k \geq 1$. O menor número natural com esta propriedade é chamado índice de nilpotência.

Destacamos que toda álgebra de Lie nilpotente é solúvel. As álgebras das matrizes triangulares superiores são exemplos de álgebras de Lie solúveis.

\subsection{Grupos Algébricos}

As definições básicas referentes à Geometria Algébrica, mencionadas nesta tese, podem ser encontrados em [16].

Seja $G$ um grupo. Denote por $\mu_{G}: G \times G \rightarrow G,(x, y) \mapsto x y$, a multiplicação do grupo e $\mathfrak{i}_{G}: G \rightarrow G, x \mapsto x^{-1}$, o inverso.

Definição 1.2.1: Seja $G$ um grupo munido com uma estrutura de variedade algébrica. Se a multiplicação de grupo $\mu_{G}$ e a inversa $\mathfrak{i}_{G}$ são morfismos de variedades algébricas, então dizemos que $G$ é um grupo algébrico. Se $G$ é uma variedade algébrica afim, dizemos que $G$ é um grupo algébrico afim.

Observação 1.2.2: Todos os grupos algébricos nesta tese serão grupos algébricos afins.

Exemplo 1.2.3: 1) $(\mathbb{R},+),(\mathbb{C},+),\left(\mathbb{R}^{*},.\right)$ são exemplos de grupos algébricos.

2) Denote por $G L(n, \mathbb{K})$ o conjunto de todas as matrizes inversíveis $n \times n$ com entradas em $\mathbb{K}$. Tem-se que $G L(n, \mathbb{K})$ é um grupo algébrico.

3) O conjunto de todas as matrizes $n \times n$ com entradas em $\mathbb{K}$ e determinante igual a 1 é um grupo algébrico, denotado por $S L(n, \mathbb{K})$. 
Definição 1.2.4: Um elemento $x \in G L(V)$ é um elemento unipotente se é a soma da identidade e um endomorfismo nilpotente. Um grupo algébrico é unipotente se todos os seus elementos são unipotentes.

O seguinte critério será útil na prova no último capítulo.

Teorema 1.2.5: Seja $G$ um grupo algébrico. Então $G$ é unipotente se, e somente se, $G /[G, G]$ é isomorfo a $(\mathbb{K},+)^{n}$ para algum $n$ natural.

Terminamos esta seção com uma observação que nos será útil quando formos calcular as álgebras de Lie correspondentes a um determinado grupo algébrico. " $X \in$

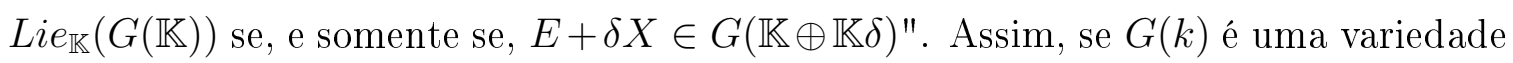
algebrica sobre $\mathbb{K}$, então $G(\mathbb{K} \oplus \mathbb{K} \delta)$ é a mesma variedade algébrica sobre $\mathbb{K} \oplus \mathbb{K} \delta$."

\subsection{Loops de Bol}

Para maiores informações sugerimos às seguintes referências bibliográficas: [3], [5], [11] e [14].

Definição 1.3.1: Um loop é um conjunto $L$, munido com uma operação binária • e um elemento identidade bilateral, denotado por 1 , tal que as equações $a \cdot x=b$ e $y \cdot a=b$ têm soluções únicas para quaisquer $a, b \in L$.

Observamos que um grupo é um loop associativo. A partir de agora, a fim de simplificar a notação, escreveremos simplesmente $a b$ ao invés de $a \cdot b$, para quaisquer elementos $a, b \in L$.

Definição 1.3.2: Seja $L$ um loop. A identidade $((x y) z) y=x((y z) y)$, para quaisquer $x, y, z \in L$, é chamada a identidade de Bol à direita. Um loop de Bol à direita é um loop que satisfaz a identidade de Bol à direita.

Nesta tese, omitiremos o termo "à direita" e escreveremos apenas "loop de Bol" uma vez que estamos interessados naqueles loops que satisfazem a identidade de Bol à direita. 
Definição 1.3.3: Um subconjunto $K$ de um grupo $G$ é chamado um subgrupo torcido se cumpre as seguintes condições:

(1) $1 \in K$,

(2) Para quaisquer $x, y \in K$ tem-se $x^{-1} \in K$ e $x y x \in K$.

Dado um loop $L$, existem duas aplicações de $L$ nele mesmo que destacaremos agora: $R_{x}: L \rightarrow L$ definida por $R_{x}(a)=a x$ e $L_{x}: L \rightarrow L$ definida por $L_{x}(a)=x a$. Segue imediatemente da definição de loop que $R_{x}$ e $L_{x}$ são permutações de $L$, para todo $x \in L$. A aplicação $R_{x}$ é chamada a multiplicação à direita e $L_{x}$ é chamada de multiplicação à esquerda.

Definição 1.3.4: Seja $L$ um loop e $K=\left\{R_{x}: x \in L\right\}$ o conjunto das multiplicações à direita de $L$, considerados como um subconjunto do grupo simétrico $\operatorname{Sym}(L)$ de $L$. Seja $G=<K>$ o subgrupo de $\operatorname{Sym}(L)$ gerado por $K$. $G$ é chamado o grupo das multiplicações à direita de $L$ e é denotado por $\operatorname{RMlt}(L)$. Seja $H=\operatorname{Stab}_{G}(1)=$ $\left\{g \in G \mid 1^{g}=1\right\}$, isto é, $H$ é o estabilizador em $G$ do elemento identidade 1 de $L$. O subgrupo $H$ é chamado o grupo das aplicações internas à direita de $L$.

Denotemos por $\operatorname{Mult}(L)$ o subgrupo de $\operatorname{Sym}(L)$ gerado pelas multiplicações à direita e à esquerda de $L$, isto é,

$$
\operatorname{Mult}(L)=<\left\{R_{x}, L_{y} \mid x, y \in L\right\}>
$$

Definição 1.3.5: Um loop $L$ é unipotente se $M u l t(L)$ é um subgrupo das matrizes triangulares superiores com 1 na diagonal.

Note que, sempre podemos identificar o loop $L$ com o subconjunto $\left\{R_{x} \mid x \in L\right\}$ de $\operatorname{RMtl}(L)$.

Definição 1.3.6: Um loop folder é uma tripla $\xi=(G, H, M)$, onde $G$ é um grupo, $H$ é um subgrupo de $G$ e $M$ é um subconjunto de $G$ contendo 1 tal que ele é um conjunto de representantes das classes laterais $H^{g}$ em $G$ para todo $g \in G$.

Em outras palavras, a definição acima nos diz que todo elemento $x \in G$ pode ser decomposto de forma única como $x=h^{g} m$, onde $h \in H$ e $m \in M$. Neste caso, defin- 
imos um produto $*: M \times M \rightarrow M$ por $m * n=k$, onde $m, n, k \in M$ e $H m n=H k$. A tripla $(G, H, M)$ é chamada um loop folder de $L=(M, *)$ e $G$ é o grupo envolvente de $L$. Para qualquer loop $L$, podemos construir um loop folder $(G, H, M)$ tal que $L \simeq(M, *)$ da seguinte forma: Seja $M$ o conjunto das multiplicações à direita $R_{x}: L \rightarrow L$ e $G=<M>$ e $H$ o estabilizador de 1 em $G$. Então $(G, H, M)$ é um loop folder.

Nosso próximo passo é definir loop de Bol algébrico. De acordo com Nagy (Tese de Doutorado) Lema 1.0.1 não é necessário exigir que as divisões à esquerda e à direita sejam morfismos de variedades algébricas. Assim tem-se a seguinte definição semelhante àquela dada para grupos algébricos.

Definição 1.3.7: Seja $L$ uma variedade algébrica sobre o corpo $\mathbb{K}$ que também é um loop de Bol. Dizemos que $L$ é um loop de Bol algébrico se a aplicação

$$
L \times L \rightarrow L(x, y) \mapsto x y^{-1}
$$

é um morfismo de variedades algébricas.

Da mesma forma que definimos loop algébrico local, podemos definir loop de Bol algébrico local.

Definição 1.3.8: Sejam $G$ um grupo algébrico sobre um corpo algebricamente fechado $\mathbb{K}, H$ um subgrupo fechado e $M$ um subconjunto fechado de $G$. Assuma que para cada conjugado $H^{g}$ de $H$ em $G$, a aplicação

$$
H^{g} \times M \rightarrow G, \quad(h, m) \mapsto h m
$$

é um morfismo biregular. Então a tripla $(G, H, M)$ é chamado um loop folder algébrico e o loop $L$ correspondente é um loop fortemente algébrico.

\section{4 Álgebras de Bol}

As definições e resultados citados nesta seção podem ser encontrados nas seguintes referências bibliográficas: [3], [6], [9] e [14]. 
Definição 1.4.1: Um espaço vetorial de dimensão finita $\mathcal{B}$, sobre $\mathbb{K}$, munido com uma operação trilinear $(\cdot ; \cdot, \cdot): \mathcal{B} \times \mathcal{B} \times \mathcal{B} \rightarrow \mathcal{B}$ é chamado um sistema triplo de Lie se valem as seguintes identidades,

$$
\begin{gathered}
(x ; y, y)=0 \\
(x ; y, z)+(y ; z, x)+(z ; x, y)=0 \\
((x ; a, b) ; y, z)+(x ;(y ; a, b), z)+(x ; y,(z ; a, b))=((x ; y, z) ; a, b)
\end{gathered}
$$

valem para quaisquer $x, y, z, a, b \in \mathcal{B}$.

A seguinte construção fornece uma infinidade de sistemas triplos de Lie (Veja [2]).

Seja $\mathcal{G}$ uma álgebra de Lie sobre o corpo $\mathbb{K}$ e $\sigma$ um automorfismo involutivo de $\mathcal{G}$. Então temos que $\mathcal{G}=\mathcal{G}^{+} \oplus \mathcal{G}^{-}$, onde $\sigma \mid \mathcal{G}^{+}=I d$ e $\sigma \mid \mathcal{G}^{-}=-I d$ e todo elemento $x \in \mathcal{G}$ pode ser escrito na forma

$$
x=\frac{1}{2}(x+\sigma x)+\frac{1}{2}(x-\sigma x),
$$

onde $\frac{1}{2}(x+\sigma x) \in \mathcal{G}^{+}$e $\frac{1}{2}(x-\sigma x) \in \mathcal{G}^{-}$. Além disso, temos as seguintes inclusões:

$$
\left[\mathcal{G}^{+}, \mathcal{G}^{+}\right] \subset \mathcal{G}^{+},\left[\mathcal{G}^{+}, \mathcal{G}^{-}\right] \subset \mathcal{G}^{-},\left[\mathcal{G}^{-}, \mathcal{G}^{-}\right] \subset \mathcal{G}^{+}
$$

Então subespaço $\mathcal{G}^{-}$torna-se um sistema triplo de Lie sob a operação

$$
(x ; y, z):=[x,[y, z]] .
$$

Vamos mostrar que a partir de um sistema triplo de Lie podemos obter uma álgebra de Lie. De fato, seja $\mathcal{B}$ um sistema triplo de Lie e defina, para $x, y \in \mathcal{B}$ fixados a seguinte aplicação:

$$
h(x, y): \mathcal{B} \rightarrow \mathcal{B}, z \mapsto(z ; x, y)
$$

Verifica-se facilmente que $h$ é uma transformação linear de $\mathcal{B}$ nele mesmo. Seja $H$ um subespaço das transformações lineares do sistema triplo de Lie $\mathcal{B}$ nele mesmo, cujos elementos são da forma $h(x, y)$. O espaço vetorial $\mathcal{G}=\mathcal{B} \oplus H$ torna-se uma álgebra de Lie do seguinte modo:

$$
[A, B]=A B-B A,[A, x]=-[x, A]=A(x),[x, y]=h(x, y),
$$


onde $A, B \in H$ e $x, y \in \mathcal{B}$.

Vamos definir a aplicação $\sigma: \mathcal{G} \rightarrow \mathcal{G}$ com a seguinte propriedade:

$$
\sigma(g)=\left\{\begin{array}{l}
g, \text { se } g \in H \\
-g, \text { se } g \in \mathcal{B}
\end{array} .\right.
$$

Então $\sigma$ é um automorfismo involutivo da álgebra de Lie $\mathcal{G}=\mathcal{B} \oplus H$.

Definição 1.4.2: A álgebra de Lie $\mathcal{G}$, construída acima, é chamada a álgebra envolvente universal do sistema triplo de Lie $\mathcal{B}$.

Definição 1.4.3: Uma derivação de um sistema triplo de Lie $\mathcal{B}$ é uma transformação linear $D: \mathcal{B} \rightarrow \mathcal{B}$ tal que

$$
D(x ; y, z)=(D x ; y, z)+(x ; D y, z)+(x ; y, D z)
$$

para quaisquer $x, y, z \in \mathcal{B}$.

Denote por $\operatorname{Der}(\mathcal{B})$ o conjunto de todas as derivações de um sistema triplo de Lie $\mathcal{B}$. Sejam $D_{1}, D_{2} \in \operatorname{Der}(\mathcal{B})$ duas derivações de $\mathcal{B}$. Então $\left[D_{1}, D_{2}\right]=D_{1} D_{2}-D_{2} D_{1}$ é uma derivação de $\mathcal{B}$. Portanto, temos que $\operatorname{Der}(\mathcal{B})$ é uma álgebra de Lie, chamada a álgebra de derivação de $\mathcal{B}$.

Se $A, B, C$ são subconjuntos de um sistema triplo de Lie $\mathcal{B}$. Por $(A ; B, C)$, entendemos o conjunto de todos os elementos da forma $\sum_{i}\left(x_{i} ; y_{i}, z_{i}\right)$, onde $x_{i} \in A, y_{i} \in B$ e $z_{i} \in C$. Observamos que as somas são finitas.

Definição 1.4.4: Seja $\mathcal{B}$ um sistema triplo de Lie sobre um corpo $\mathbb{K}$. Um subespaço $\mathcal{D}$ de $\mathcal{B}$ é um subsistema de $\mathcal{B}$ se $(\mathcal{D} ; \mathcal{D}, \mathcal{D}) \subset \mathcal{D}$ e, é chamado um ideal de $\mathcal{B}$ se $(\mathcal{B} ; \mathcal{B}, \mathcal{D})=\mathcal{D}$.

Seja $\mathcal{D}$ um ideal de um sistema triplo de Lie $\mathcal{B}$ e definimos indutivamente os seguintes subconjuntos de $\mathcal{B}: \mathcal{D}^{(0)}=\mathcal{D}, \mathcal{D}^{(1)}=(\mathcal{D} ; \mathcal{D}, \mathcal{B})$ e $\mathcal{D}^{(k)}=\left(\mathcal{D}^{(k-1)} ; \mathcal{D}^{(k-1)}, \mathcal{B}\right)$, para todo número natural $k$ maior ou igual a 1 . Em [9] prova-se que para todo $k \in \mathbb{N}$, o subespaço $\mathcal{D}^{(k)}$ é um ideal de $\mathcal{D}^{(k-1)}$ e, portanto temos a seguinte cadeia de ideais:

$$
\mathcal{D} \supseteq \mathcal{D}^{(1)} \supseteq \mathcal{D}^{(2)} \supseteq \cdots \supseteq \mathcal{D}^{(k)} \supseteq \cdots
$$


Definição 1.4.5: Um ideal $\mathfrak{D}$ de um sistema triplo de Lie é solúvel se existe um número natural $k$ tal que $\mathcal{D}^{(k)}=0$.

Provou-se em [9] que todo sistema triplo de Lie possui um único ideal maximal solúvel, que é denotado por $\mathcal{R}(\mathcal{B})$ e é chamado o radical de $\mathcal{B}$. Um sistema triplo de Lie $\mathcal{B}$ é semi-simples se $\mathcal{R}(\mathcal{B})=0$.

Proposição 1.4.6: 1) A álgebra envolvente de um sistema triplo de Lie solúvel é solúvel. Se um sistema triplo de Lie tem alguma álgebra de Lie envolvente solúvel, então ele é solúvel.

2) Se $\mathcal{B}$ é um sistema triplo de Lie semi-simples então sua álgebra de Lie envolvente universal também é semi-simples.

Definição 1.4.7: Um espaço vetorial $\mathcal{B}$, sobre um corpo $\mathbb{K}$, é chamado uma álgebra de Bol se ela tem uma operação binária $(\cdot, \cdot)$ e uma operação trilinear $(\cdot ; \cdot, \cdot)$ que são lineares em todas as variáveis e valem as seguintes identidades,

$$
\begin{gathered}
(x ; y, y)=0 . \\
(x ; y, z)+(y ; z, x)+(z ; x, y)=0 . \\
((x ; y, z) ; u, v)=((x ; u, v) ; y, z)+(x ;(y ; u, v), z)+(x ; y,(z ; u, v)) . \\
x \cdot x=0 . \\
(x \cdot y ; u, v)=(x ; u, v) \cdot y+x \cdot(y ; u, v)+(u \cdot v ; x, y)+(x \cdot y) \cdot(u \cdot v)
\end{gathered}
$$

para quaisquer $x, y, z, u, v \in \mathcal{B}$.

Observação 1.4.8: 1) As identidades (1.4),(1.5), (1.6) significam que uma álgebra de Bol é um sistema triplo de Lie com respeito à operação trilinear $(\cdot ; \cdot, \cdot)$. Em particular, todo sistema triplo de Lie pode ser considerado como uma álgebra de Bol com a multiplicação trivial, isto é, $x \cdot y=0$ para quaisquer $x, y \in \mathcal{B}$.

2) Para quaisquer $x, y \in \mathcal{B}$ vale $x \cdot y=-y \cdot x$.

3) Para quaisquer $x, y, z \in \mathcal{B}$ vale $(x ; y, z)=-(x ; z, y)$. De fato, $0=(x ; y+z, y+z)=$ $(x ; y, z)+(x ; z, y)$ de onde obtemos $(x ; y, z)=-(x ; z, y)$.

4) A partir de agora escreveremos simplesmente $x y$ ao invés de $x \cdot y$. 
Definição 1.4.9: Um subconjunto $\mathcal{I}$ de uma álgebra de Bol $\mathcal{B}$ é um ideal se $(\mathcal{B} ; \mathcal{B}, \mathcal{I})$ e $\mathcal{B I} \subset \mathcal{I}$.

Seja $\mathcal{B}$ uma álgebra de Bol e $\mathcal{I}$ um ideal de $\mathcal{B}$. No conjunto

$$
\mathcal{B} / \mathcal{I}=\{x+\mathcal{I} \mid x \in \mathcal{B}\}
$$

podemos definir duas operações. Dados $x+\mathcal{I}, y+\mathcal{I}, z+\mathcal{I} \in \mathcal{B}+\mathcal{I}$ definimos

$$
(x+\mathcal{I})(y+\mathcal{I})=x y+\mathcal{I} \text { e }(x+\mathcal{I} ; y+\mathcal{I}, z+\mathcal{I})=(x ; y, z)+\mathcal{I} .
$$

Estas operações não dependem dos representantes e, portanto $\mathcal{B} / \mathcal{I}$ é uma álgebra de Bol, chamada álgebra de Bol quociente de $\mathcal{B}$ por $\mathcal{I}$.

Definição 1.4.10: Seja $\mathcal{B}$ uma álgebra de Bol. Definimos o centro de $\mathcal{B}$, denotado por $\mathcal{Z}(\mathcal{B})$, pelo conjunto

$$
\{x \in \mathcal{B} \mid x \cdot y=(x ; y, z)=(y ; x, z)=(y ; z, x)=0, \text { para quaisquer } y, z \in \mathcal{B}\} \text {. }
$$

Observação 1.4.11: A definição acima é equivalente a $\{x \in \mathcal{B} \mid x \cdot y=(x ; y, z)=$ $(y ; x, z)=0$, para quaisquer $y, z \in \mathcal{B}\}$. De fato, pelo item 3$)$ da observação acima, temos que $(y ; x, z)=0$ se, e somente se, $(y ; z, x)=0$. A partir de agora, quando falarmos em centro de uma álgebra de Bol, estaremos nos referindo a este conjunto, isto é, $\mathcal{Z}(\mathcal{B})=\{x \in \mathcal{B} \mid x \cdot y=(x ; y, z)=(y ; x, z)=0$, para quaisquer $y, z \in \mathcal{B}\}$.

Lema 1.4.12: Seja $\mathcal{B}$ uma álgebra de Bol. Então $\mathcal{Z}(\mathcal{B})$ é um ideal de $\mathcal{B}$.

Seja $\mathcal{I}$ um ideal de uma álgebra de Bol $\mathcal{B}$. Definimos indutivamente aos seguintes subconjuntos de $\mathcal{B}$.

$\mathcal{I}^{(0)}=\mathcal{I}, \mathcal{I}^{(s+1)}=\mathcal{I}^{(s)} \mathcal{I}^{(s)}+\left(\mathcal{I}^{(s)} ; \mathcal{I}^{(s)}, \mathcal{B}\right)$ e $\mathcal{B}^{(0)}=\mathcal{B}, \mathcal{B}^{(s+1)}=\mathcal{B}^{(s)} \mathcal{B}^{(s)}+\left(\mathcal{B}^{(s)} ; \mathcal{B}^{(s)}, \mathcal{B}\right)$ para todo $s>0$.

Lema 1.4.13: $[9]$ Para todo $k>0, \mathcal{I}^{(k)}$ é um ideal de $\mathcal{I}^{(k-1)}$. Assim, temos

$$
\mathcal{I}=\mathcal{I}^{(0)} \triangleright \mathcal{I}^{(1)} \triangleright \mathcal{I}^{(2)} \triangleright \cdots .
$$


Definição 1.4.14: Um ideal $\mathcal{I}$ de uma álgebra de Bol $\mathcal{B}$ é solúvel se $\mathcal{I}^{(k)}=0$ para algum $k \geq 0$. Uma álgebra de $\operatorname{Bol} \mathcal{B}$ é solúvel se $\mathcal{B}^{(k)}=0$, para algum $k \geq 0$.

Sejam $\mathcal{B}$ uma álgebra de Bol e $l>1$ um número inteiro. Definimos os seguintes subconjuntos de $\mathcal{B}$.

$$
\mathcal{B}^{1}=\mathcal{B}, \mathcal{B}^{l}=\mathcal{B B}^{l-1}+\sum_{i+j+k=l}\left(\mathcal{B}^{i} ; \mathcal{B}^{j}, \mathcal{B}^{k}\right)
$$

Definição 1.4.15: Uma álgebra de $\operatorname{Bol} \mathcal{B}$ é nilpotente se existe $k>1$ tal que $\mathcal{B}^{k}=$ 0. O menor número com a propriedade acima é chamado de índice de nilpotência.

Lema 1.4.16: Para todo $k>1, \mathcal{B}^{k}$ é um ideal de $\mathcal{B}^{k-1}$.

Lema 1.4.17: Seja $\mathcal{B}$ uma álgebra de Bol nilpotente. Então $\mathcal{Z}(\mathcal{B})$ não é trivial.

Demonstração: Basta lembrar que $B^{l}=0$ se, e somente se, $\mathcal{B B}^{l-1}=\{0\}$ e $\left(\mathcal{B}^{i} ; \mathcal{B}^{j}, \mathcal{B}^{k}\right)=\{0\}$ para quaisquer $i, j, k$ tais que $i+j+k=l$.

Definição 1.4.18: Sejam $\mathcal{B}$ e $\mathcal{B}^{\prime}$ duas álgebras de Bol sobre o corpo $\mathbb{K}$. Uma aplicação linear $T: \mathcal{B} \rightarrow \mathcal{B}^{\prime}$ é um homomorfismo de álgebras de Bol se valem as seguintes igualdades,

1) $T(x y)=(T x)(T y)$,

2) $T(x, y, z)=(T x, T y, T z)$

para quaisquer $x, y, z \in \mathcal{B}$.

Se $T: \mathcal{B} \rightarrow \mathcal{B}^{\prime}$ é um isomorfismo de espaços vetoriais que também é um homomorfismo de álgebras de Bol, então dizemos que $T$ é um isomorfismo de álgebras de Bol .

Definição 1.4.19: Um endomorfismo linear de uma álgebra de Bol $\mathcal{B}$ é uma pseudoderivação com companheiro $z \in \mathcal{B}$ se valem as seguintes identidades:

1) $D(x y)=D(x) y+x D(y)+(z ; x, y)+(x y) z$.

2) $D(x ; y, z)=(D x ; y, z)+(x ; D y, z)+(x ; y, D z)$ para quaisquer $x, y, z \in \mathcal{B}$. 
Lema 1.4.20: $\mathrm{O}$ conjunto de todas as pseudoderivações de uma álgebra de Bol $\mathcal{B}$ constituem uma álgebra de Lie com respeito às operações naturais de adição, multiplicação por escalar e o colchete de Lie definido por

$$
\left[D_{1}, D_{2}\right]=D_{1} D_{2}-D_{2} D_{1}
$$

onde $D_{1}$ e $D_{2}$ são pseudoderivações quaisquer da álgebra de Bol $\mathcal{B}$.

Denotamos a álgebra de Lie das pseudoderivações de uma álgebra de Bol $\mathcal{B}$ por pder $\mathcal{B}$.

Lema 1.4.21: Sejam $D_{1}$ e $D_{2}$ duas pseudoderivações quaisquer da álgebra de Bol $\mathcal{B}$, com companheiros $k_{1}$ e $k_{2}$, respectivamente. Então:

(1) $D_{1}+D_{2}$ é uma pseudoderivação de $B$ com companheiro $k_{1}+k_{2}$.

(2) Se $\lambda \in \mathbb{K}$, então $\lambda D_{1}$ é uma pseudoderivação de $\mathcal{B}$ com companheiro $\lambda k_{1}$.

(3) $\left[D_{1}, D_{2}\right]$ é uma pseudoderivação de $\mathcal{B}$ com companheiro $k_{1} k_{2}+D_{1} k_{2}-D_{2} k_{1}$.

Observação 1.4.22: 1) Notemos que uma pseudoderivação pode ter mais de um companheiro.

2) Se $D$ é uma pseudoderivação de uma álgebra de $\operatorname{Bol} \mathcal{B}$, denotaremos o conjunto de todos os companheiros de $D$ por $\operatorname{com}(D)$.

Lema 1.4.23: Seja $\mathcal{B}$ uma álgebra de Bol. Então para quaisquer pseudoderivações $D_{1}, D_{2} \in \operatorname{pder}(\mathcal{B})$ e $\lambda \in \mathbb{K}$ temos:

(1) $\operatorname{com}\left(D_{1}\right)+\operatorname{com}\left(D_{2}\right) \subseteq \operatorname{com}\left(D_{1}+D_{2}\right)$;

(2) $\lambda \operatorname{com}\left(D_{1}\right) \subseteq \operatorname{com}\left(\lambda D_{1}\right)$;

(3) Se $a \in \operatorname{com}\left(D_{1}\right), b \in \operatorname{com}\left(D_{2}\right)$, então $a \cdot b+D_{1} b-D_{2} a \in \operatorname{com}\left(\left[D_{1}, D_{2}\right]\right)$.

Lema 1.4.24: $\mathrm{O}$ conjunto $\operatorname{Pder}(\mathcal{B})=\{(D, a) ; D \in \operatorname{pder}(\mathcal{B}), a \in \operatorname{com}(D)\}$, munido com as operações

(i) $\left(D_{1}, a_{1}\right)+\left(D_{2}, a_{2}\right)=\left(D_{1}+D_{2}, a_{1}+a_{2}\right)$.

(ii) $\lambda\left(D_{1}, a_{1}\right)=\left(\lambda D_{1}, a_{1}\right)$.

(iii) $\left[\left(D_{1}, a_{1}\right),\left(D_{2}, a_{2}\right)\right]=\left(\left[D_{1}, D_{2}\right], a_{1} a_{2}+D_{1} a_{2}-D_{2} a_{1}\right)$

é uma álgebra de Lie. 
Definição 1.4.25: Seja $\mathcal{B}$ uma álgebra de Bol. A álgebra de Lie $\operatorname{Pder}(\mathcal{B})$, definida no Lema acima, é chamada a álgebra ampliada das pseudoderivações da álgebra de Bol $\mathcal{B}$.

Lembramos que, por definição, $D_{x, y}$ é uma pseudoderivação da álgebra de $\operatorname{Bol} \mathcal{B}$, para quaisquer $x, y \in \mathcal{B}$, onde $D_{x, y}(z)=(z ; x, y)$.

Definição 1.4.26: Seja $\mathcal{B}$ uma álgebra de Bol. $O$ conjunto de todas as pseudoderivações de $\mathcal{B}$, geradas por $\left\{D_{x, y}: x, y \in \mathcal{B}\right\}$, é chamado o conjunto das pseudoderivações internas da álgebra de $\operatorname{Bol} \mathcal{B}$ e é denotado por iper $(\mathcal{B})$.

Note que $\operatorname{ipder}(\mathcal{B})$ é uma subálgebra da álgebra de Lie $\operatorname{pder}(\mathcal{B})$. Assim sendo, $\operatorname{Ipder}(\mathcal{B})$ pode ser introduzida como o conjunto de todos os pares $(D, a)$, onde $D \in$ $\operatorname{ipder}(\mathcal{B}), a \in \operatorname{com}(D)$. Naturalmente, $\operatorname{Ipder}(\mathcal{B})$ é uma subálgebra da álgebra de Lie $\operatorname{Pder}(\mathcal{B})$.

Definição 1.4.27: A álgebra de Lie $\operatorname{Ipder}(\mathcal{B})$ é chamada a álgebra ampliada das pseudoderivações internas de $\mathcal{B}$.

Definição 1.4.28: Seja $\mathcal{B}$ uma álgebra de Bol, $K$ uma subálgebra de $\operatorname{Pder}(\mathcal{B})$ tal que $\operatorname{Ipder}(\mathcal{B}) \subset K$. Sobre a soma direta $B \oplus K$ definimos uma estrutura de álgebra de Lie, pela regra:

(1) $[x, y]=-\left(D_{x, y}, x y\right)$,

(2) $[x,(D, a)]=-D(x)$ e $[(D, a), x]=D(x)$,

(3) $\left[\left(D_{1}, a_{1}\right),\left(D_{2}, a_{2}\right)\right]=\left(\left[D_{1}, D_{2}\right], a_{1} a_{2}+D_{1} a_{2}-D_{2} a_{1}\right)$

para quaisquer $x, y, a, a_{1}, a_{2} \in \mathcal{B}$.

Qualquer álgebra definida desta maneira é chamada álgebra de Lie envolvente da álgebra de Bol $\mathcal{B}$, e é denotada por $\operatorname{Env}(\mathcal{B})$.

Definição 1.4.29: Tomando $K=\operatorname{Pder}(\mathcal{B})$ obtemos a álgebra de Lie envolvente maximal e tomando $K=\operatorname{Ipder}(\mathcal{B})$, obtemos a álgebra de Lie envolvente minimal. 
Neste trabalho, sempre consideraremos $K=\operatorname{Ipder}(\mathcal{B})$ e a partir de agora diremos simplesmente álgebra de Lie envolvente omitindo o adjetivo "minimal".

Proposição 1.4.30: Para qualquer álgebra de Bol $\mathcal{B}$ existe um par de álgebras de Lie $(\mathcal{G}, \mathcal{H})$ tal que $\mathcal{G}=\mathcal{M} \oplus \mathcal{H},[\mathcal{M},[\mathcal{M}, \mathcal{M}]] \subset \mathcal{M},[\mathcal{M}, \mathcal{M}] \cap \mathcal{M}=\{0\}, \operatorname{dim}(\mathcal{M})=$ $\operatorname{dim}(\mathcal{B})$ tal que

$$
X Y=\operatorname{pr}[X, Y] \text { e }[Z,[X, Y]]=(Z ; X, Y)
$$

define em $\mathcal{M}$ uma álgebra de Bol isomorfa a álgebra de Bol $\mathcal{B}$.

Proposição 1.4.31: Para qualquer par de álgebras de Lie $(\mathcal{G}, \mathcal{H})$ tal que $\mathcal{G}=\mathcal{M} \oplus$ $\mathcal{H},[\mathcal{M},[\mathcal{M}, \mathcal{M}]] \subset \mathcal{M},[\mathcal{M}, \mathcal{M}] \cap \mathcal{M}=\{0\}$, define em $\mathcal{M}$ uma estrutura de álgebra de Bol com as operações

$$
\operatorname{pr}[X, Y]=X Y \text { e }(Z ; X, Y)=[Z,[X, Y]]
$$

para quaisquer $X, Y, Z \in \mathcal{M}$.

Observação 1.4.32: Na verdade, a álgebra de Lie $\mathcal{G}=\mathcal{M} \oplus \mathcal{H}$, considerada na proposição anterior, coincide com alguma álgebra de Lie envolvente da álgebra de Bol obtida precisamente a menos de isomorfismos.

\section{5 Álgebra tangente de um loop de Bol}

Definição 1.5.1: Um grupo de Lie local é uma variedade diferenciável U junto com um ponto base $e$, uma vizinhança $V$ de $e$ e uma aplicação diferenciável $\mu$ : $V \times V \rightarrow U, \quad(x, y) \mapsto x y$ satisfazendo as condições $e x=x e=x$ e $(x y) z=x(y z)$ para todo $x, y, z, x y, y z \in V$. Um loop analítico é uma variedade analítica sobre $\mathbb{R}$ ou $\mathbb{C}$.

Da mesma forma que definimos um grupo de Lie local, podemos definir loop analítico local.

Definição 1.5.2: Uma álgebra tangente $\mathcal{G}$ de um loop analítico local $L$ é uma álgebra munida de uma operação bilinear $[\cdot, \cdot]$ e uma operação trilinear $(\cdot, \cdot, \cdot)$. 
Observação 1.5.3: Se $G$ é um grupo de Lie local, então $(x, y, z)=0$ para todo $x, y, z \in \mathcal{G}$. Além disso, no caso geral, $[x, x]=0$ para todo $x \in \mathcal{G}$.

O próximo teorema caracteriza a álgebra tangente de um loop de Bol analítico.

Teorema 1.5.4: A álgebra tangente de um loop de Bol analítico local é uma álgebra de Bol. Toda álgebra de Bol de dimensão finita é isomórfica a uma álgebra tangente, a menos de isomorfismo, de um loop de Bol analítico local. 


\section{Capítulo 2}

\section{Álgebras de Bol de dimensão 2}

\section{1 Álgebras de Bol de dimensão 2 sobre o corpo algebricamente fechado}

Nesta seção, queremos classificar, a menos de isomorfismo, todas as álgebras de Bol $\mathcal{B}$ de dimensão 2 sobre um corpo algebricamente fechado $\mathbb{K}$ de característica 0 .

Caso (I): Existem $x, y \in \mathcal{B}$ tais que $x y \neq 0$.

Como a operação · é anti-simétrica, existe uma base $\{v, w\}$ de $\mathcal{B}$ tal que

$$
v w=v \text { e } w v=-v
$$

e, consequentemente temos

$$
v v=w w=0 .
$$

Por fim, queremos definir em $\mathcal{B}$ uma estrutura de sistema triplo de Lie, isto é, definir $(\cdot ; \cdot, \cdot): \mathcal{B} \times \mathcal{B} \times \mathcal{B} \rightarrow \mathcal{B}$ de modo que as identidades (1.1), (1.2), (1.3) sejam satifeitas.

A fim de que a identidade (1.1) seja satisfeita, devemos definir $(\cdot, \cdot, \cdot)$, nas triplas $(v ; w, w),(w ; v, v)$, da seguinte maneira:

$$
(v ; w, w)=(w ; v, v)=0
$$

Temos da identidade (1.2) as seguintes relações:

$$
(w ; v, w)=-(v ; w, w)-(w ; w, v)=-(w ; w, v)
$$




$$
(v ; w, v)=-(w ; v, v)-(v ; v, w)=-(v ; v, w)
$$

Supomos agora

$$
(v ; v, w)=\alpha v+\beta w=-(v ; w, v)
$$

e

$$
(w ; w, v)=\gamma v+\theta w=-(w ; v, w)
$$

onde $\alpha, \beta, \gamma, \theta$ são escalares em $\mathbb{K}$. Nosso objetivo é determinar $\alpha, \beta, \gamma$ e $\theta \in \mathbb{K}$ a partir das identidades (1.3) e (1.8).

A seguinte observação irá eliminar alguns cálculos no que segue.

Observação 2.1.1: 1) Numa álgebra de $\operatorname{Bol} \mathcal{B}$, temos que $(a b)(x y)=0$, para quaisquer $a, b, x, y \in \mathcal{B}$. Assim, a identidade (1.8) pode ser escrita simplesmente como

$$
(a b ; x, y)=(a ; x, y) b+a(b ; x, y)+(x y ; a, b)
$$

para quaisquer $a, b, x, y \in \mathcal{B}$.

3) Da identidade (2.6) para $a=x=v$ e $b=y=w$

$$
(v w ; v, w)=(v ; v, w) w+v(w ; v, w)+(v w ; v, w)
$$

ou seja, se, e somente se,

$$
(\alpha v+\beta w) w-v(\gamma v+\theta w)=0
$$

isto é, se, e somente se,

$$
(\alpha-\theta) v=0,
$$

cuja igualdade vale se, e somente se, $\alpha=\theta$.

Portanto as equações (2.5) e (2.6) podem ser escritas do seguinte modo:

$$
(v, v, w)=\alpha v+\beta w=-(v, w, v)
$$

e

$$
(w, w, v)=\gamma v+\alpha w=-(w, v, w) .
$$


Mostraremos que os parâmetros $\alpha, \beta$ e $\gamma$ não podem ser obtidos a partir dos outros dois, mostrando que as equações (1.3) e (1.8) são satisfeitas desde que as triplas sejam definidas de acordo com as equações (2.7) e (2.8) . Para isto, vamos usar o lema abaixo cuja demostração é uma consequência das definições de álgebra de Bol.

Lema 2.1.2: Seja $\mathcal{B}$ uma álgebra de Bol de dimensão 2 sobre o corpo $\mathbb{K}$ e base $\{v, w\}$ que satisfaz (2.1) e a aplicação ternária definida como (2.8) e (2.9). Então são verdadeiras as seguintes igualdades: $(1)(a b ; x, y)=(x y ; a, b)$,

$(2)(a ; x, y) b+a(b ; x, y)=0$,

$(3)((x ; a, b) ; y, z)=((x ; y, z) ; a, b)$,

(4) $(x ;(y ; a, b), z)+(x ; y,(z ; a, b))=0$,

onde as equações (1) e (2) provém da identidade (2.6), e as equações (3), (4) provém da identidade (1.3).

Em vista do exposto acima, podemos enunciar o seguinte resultado.

Teorema 2.1.3: Seja $\mathcal{B}$ um espaço vetorial de dimensão 2 e $(\alpha, \beta, \gamma) \in \mathbb{K}$. Então existe uma base $\{v, w\}$ de $\mathcal{B}$ tal que com a operação bilinear anti-simétrica

$$
\because: \mathcal{B} \times \mathcal{B} \rightarrow \mathcal{B}
$$

definida por $v w=v$ e com a operação trilinear

$$
(\cdot ; \cdot, \cdot): \mathcal{B} \times \mathcal{B} \times \mathcal{B} \rightarrow \mathcal{B}
$$

definida por $(v ; v, w)=\alpha v+\beta w$ e $(w ; w, v)=\gamma v+\alpha w$ e (1.4) torna-se uma álgebra de Bol à direita sobre $\mathbb{K}$.

Notação: A álgebra de Bol, obtida no Teorema (2.1.13), será denotada por $\mathcal{B}(\alpha, \beta, \gamma)$.

Nosso próximo passo é classificar todas as álgebras de Bol, a menos de isomorfismos, isto é, se $\mathcal{B}(\alpha, \beta, \gamma) \simeq \mathcal{B}\left(\alpha^{\prime}, \beta^{\prime}, \gamma^{\prime}\right)$, quais são as relações que devem existir entre as triplas de escalares $(\alpha, \beta, \gamma)$ e $\left(\alpha^{\prime}, \beta^{\prime}, \gamma^{\prime}\right)$. O próximo resultado responde completamente esta questão. 
Teorema 2.1.4: Sejam $B=\{v, w\}$ uma base de $\mathcal{B}(\alpha, \beta, \gamma)$ tal que $v w=v, B^{\prime}=$ $\{x, y\}$ uma base de $\mathcal{B}\left(\alpha^{\prime}, \beta^{\prime}, \gamma^{\prime}\right)$ tal que $x y=x$ e seja

$$
T: \mathcal{B}(\alpha, \beta, \gamma) \rightarrow \mathcal{B}\left(\alpha^{\prime}, \beta^{\prime}, \gamma^{\prime}\right)
$$

uma aplicação linear definida por $T v=a x+b y$ e $T w=c x+d y$. Então $T$ : $\mathcal{B}(\alpha, \beta, \gamma) \rightarrow \mathcal{B}\left(\alpha^{\prime}, \beta^{\prime}, \gamma^{\prime}\right)$ é um isomorfismo de álgebras de Bol se, e somente se, as seguintes condições são satisfeitas:

(i) $\beta=a^{2} \beta^{\prime}$,

(ii) $\alpha=a\left(\alpha^{\prime}-c \beta^{\prime}\right)$,

(iii) $\gamma=\gamma^{\prime}-c\left(2 \alpha^{\prime}-c \beta^{\prime}\right)$,

(iv) $b=0$ e $d=1$.

Demonstração: Primeiramente, assumimos que $T$ é um homomorfismo de álgebras de Bol. Temos

$$
\begin{aligned}
a x+b y=T v=T(v w) & =(T v T w) \\
& =(a x+b y)(c x+d y)=(a d-b c) x,
\end{aligned}
$$

donde obtemos que $b=0$ e $a=a d-b c$. $\operatorname{Logo} a=a d$. Como $T$ é um isomorfismo e $b=0$, devemos ter $T v \neq 0$, isto é, $a \neq 0$. Consequentemente $d=1$ e isto prova o item $(i v)$.

Agora note que

$$
\begin{aligned}
T(v ; v, w) & =T(\alpha v+\beta w)=\alpha T v+\beta T w \\
& =(\alpha a) x+\beta(c x+y)=(a \alpha+c \beta) x+\beta y
\end{aligned}
$$

e

$$
\begin{aligned}
(T v ; T v, T w) & =(a x ; a x, c x+y)=a^{2}(x ; x, y) \\
& =a^{2}\left(\alpha^{\prime} x+\beta^{\prime} y\right)=\left(a^{2} \alpha^{\prime}\right) x+\left(a^{2} \beta^{\prime}\right) y .
\end{aligned}
$$

Portanto, $T(v ; v, w)=(T v ; T v, T w)$ se, e somente se, $\beta=a^{2} \beta^{\prime}$ e $a \alpha+c \beta=a^{2} \alpha^{\prime}$. Como $\beta=a^{2} \beta^{\prime}$, temos que $a \alpha+c \beta=a^{2} \alpha^{\prime}$ se, e somente se, $a \alpha+c\left(a^{2} \beta^{\prime}\right)=a^{2} \alpha^{\prime}$, que é equivalente a $\alpha=a\left(\alpha^{\prime}-c \beta^{\prime}\right)$. Isto prova $(i)$ e $(i i)$. 
Finalmente, por um lado temos

$$
\begin{aligned}
T(w ; w, v) & =T(\gamma v+\alpha w)=\gamma T v+\alpha T w \\
& =(\gamma a) x+\alpha(c x+y)=(a \gamma+c \alpha) x+\alpha y,
\end{aligned}
$$

e por outro lado temos,

$$
\begin{aligned}
(T w ; T w, T v) & =(c x+y ; c x+y, a x)=a c(x ; y, x)+a(y ; y, x) \\
& =-a c\left(\alpha^{\prime} x+\beta^{\prime} y\right)+a\left(\gamma^{\prime} x+\alpha^{\prime} y\right) \\
& =\left(-a c \alpha^{\prime}+a \gamma^{\prime}\right) x+\left(-a c \beta^{\prime}+a \alpha^{\prime}\right) y .
\end{aligned}
$$

Logo, $T(v ; v, w)=(T v ; T v, T w)$ se, e somente se, $\alpha=a\left(\alpha^{\prime}-c \beta^{\prime}\right)$ e $a \gamma+c \alpha=$ $-a c \alpha^{\prime}+a \gamma^{\prime}$, ou equivalentemente, $a \gamma+c\left(a\left(\alpha^{\prime}-c \beta^{\prime}\right)\right)=-a c \alpha^{\prime}+a \gamma^{\prime}$, o que nos dá $\gamma=\gamma^{\prime}-c\left(2 \alpha^{\prime}-c \beta^{\prime}\right)$, e isto prova o item (iii).

Suponha que as triplas $(\alpha, \beta, \gamma),\left(\alpha^{\prime}, \beta^{\prime}, \gamma^{\prime}\right)$ satisfaçam as relações $(i),(i i),(i i i)$. Então a transformação linear $T: \mathcal{B} \rightarrow \mathcal{B}$ definida por

$$
T v=a x \text { e } T w=c x+y
$$

é um isomorfismo entre as álgebras de $\operatorname{Bol} \mathcal{B}(\alpha, \beta, \gamma)$ e $\mathcal{B}\left(\alpha^{\prime}, \beta^{\prime}, \gamma^{\prime}\right)$.

O próximo resultado é apenas um modo ligeiramente diferente de escrever o Teorema (2.1.4).

Corolário 2.1.5: Duas álgebras de $\operatorname{Bol} \mathcal{B}(\alpha, \beta, \gamma)$ e $\mathcal{B}\left(\alpha^{\prime}, \beta^{\prime}, \gamma^{\prime}\right)$ são isomorfas se, e somente se, existem escalares $a, c \in \mathbb{K}, a \neq 0$, tais que as seguintes relações são satisfeitas

(i) $\beta=a^{2} \beta^{\prime}$,

(ii) $\alpha=a\left(\alpha^{\prime}-c \beta^{\prime}\right)$,

(iii) $\gamma=\gamma^{\prime}-c\left(2 \alpha^{\prime}-c \beta^{\prime}\right)$.

No próximo resultado consideraremos o caso particular quando $\beta=0$.

Corolário 2.1.6: $\operatorname{Sejam~} \mathcal{B}(\alpha, \beta, \gamma)$ e $\mathcal{B}\left(\alpha^{\prime}, \beta^{\prime}, \gamma^{\prime}\right)$ duas álgebras de Bol isomorfas. Então: 
(i) $\beta=0$ se, e somente se, $\beta^{\prime}=0$. Neste caso, temos $\alpha=a \alpha^{\prime}$ e $\gamma=\gamma^{\prime}-(2 c) \alpha^{\prime}$.

(ii) Suponha que $\beta=0$. Então $\alpha=0$ se, e somente se, $\alpha^{\prime}=0$.

Demonstração: Suponha que $\mathcal{B}(\alpha, \beta, \gamma)$ e $\mathcal{B}\left(\alpha^{\prime}, \beta^{\prime}, \gamma^{\prime}\right)$ duas álgebras de Bol isomorfas. Pelo Corolário (2.1.5), existem escalares $a, c \in \mathbb{K}, a \neq 0$, tal que as relações (i), (ii) e (iii) são satisfeitas.

(i) Da relação (i) temos que $\beta=0$ se, e somente se, $\beta^{\prime}=0$.

(ii) Supondo que $\beta=0$ temos da relação (ii) que $\alpha=a \alpha^{\prime}$. Portanto, desde que $a \neq 0, \alpha=0$ se, e somente se, $\alpha^{\prime}=0$.

Corolário 2.1.7: Suponha $\beta^{\prime} \neq 0$. A aplicação $T: \mathcal{B}(0,1, \gamma) \rightarrow \mathcal{B}\left(\alpha^{\prime}, \beta^{\prime}, \gamma^{\prime}\right)$ dada por $T(v)=\frac{1}{\sqrt{\beta^{\prime}}} x$ e $T(w)=\frac{\alpha^{\prime}}{\beta^{\prime}} x+y$ é um isomorfismo de álgebras de Bol, onde $\gamma=\gamma^{\prime}-\frac{\left(\alpha^{\prime}\right)^{2}}{\beta^{\prime}}$.

Demonstração: Vamos verificar as relações (i), (ii) e (iii) do Corolário 2.1 .5 com $a=\frac{1}{\sqrt{\beta^{\prime}}}, c=\frac{\alpha^{\prime}}{\beta^{\prime}}$.

(i) $a^{2} \beta^{\prime}=\left(\frac{1}{\sqrt{\beta^{\prime}}}\right)^{2} \beta^{\prime}=\frac{1}{\beta^{\prime}} \beta^{\prime}=1$;

(ii) $a\left(\alpha^{\prime}-c \beta^{\prime}\right)=a\left(\alpha^{\prime}-\frac{\alpha^{\prime}}{\beta^{\prime}} \beta^{\prime}\right)=a\left(\alpha^{\prime}-\alpha^{\prime}\right)=0$;

(iii) $\gamma^{\prime}-c\left(2 \alpha^{\prime}-c \beta^{\prime}\right)=\gamma^{\prime}-\frac{\alpha^{\prime}}{\beta^{\prime}}\left(2 \alpha^{\prime}-\frac{\alpha^{\prime}}{\beta^{\prime}} \beta^{\prime}\right)=\gamma^{\prime}-\frac{\left(\alpha^{\prime}\right)^{2}}{\beta^{\prime}}$. Portanto, as álgebras de Bol $\mathcal{B}(0,1, \gamma)$ e $\mathcal{B}\left(\alpha^{\prime}, \beta^{\prime}, \gamma^{\prime}\right)$ são isomorfas.

Corolário 2.1.8: As álgebras de $\operatorname{Bol} \mathcal{B}(0,1, \gamma)$ e $\mathcal{B}\left(0,1, \gamma^{\prime}\right)$ são isomorfas se, e somente se, $\gamma=\gamma^{\prime}$.

Demonstração: $\quad(\Rightarrow)$ Suponha que as álgebras de Bol $\mathcal{B}(0,1, \gamma)$ e $\mathcal{B}\left(0,1, \gamma^{\prime}\right)$ são isomorfas, então pelo Corolário (2.1.5) existem escalares $a, c \in \mathbb{K}, a \neq 0$ tal que as relações (i), (ii) e (iii) são satisfeitas. De (ii) temos $0=a(0-c)=a c$ e desde que $a \neq 0$ segue que $c=0$ e substituindo em (iii) concluímos que $\gamma=\gamma^{\prime}$.

A recíproca é imediata, desde que a aplicação identidade é um isomorfismo de álgebras de Bol.

Corolário 2.1.9: 1) As álgebras de $\operatorname{Bol} \mathcal{B}(\alpha, \beta, \gamma)$ e $\mathcal{B}\left(\alpha^{\prime}, \beta^{\prime}, \gamma^{\prime}\right)$, onde $\beta, \beta^{\prime} \in \mathbb{K}^{*}$, 
são isomorfas se, e somente se,

$$
\gamma-\frac{\alpha^{2}}{\beta}=\gamma^{\prime}-\frac{\alpha^{\prime 2}}{\beta^{\prime}}
$$

Nestas condições, o conjunto $\operatorname{IsO}_{\mathbb{K}}\left(\mathcal{B}(\alpha, \beta, \gamma), \mathcal{B}\left(\alpha^{\prime}, \beta^{\prime}, \gamma^{\prime}\right)\right)$, tem apenas dois elementos, a saber,

$$
T_{1} v=\sqrt{\frac{\beta}{\beta^{\prime}}} v, \quad T_{1} w=\frac{1}{\beta^{\prime}}\left(\alpha^{\prime}-\alpha \sqrt{\frac{\beta}{\beta^{\prime}}}\right) v+w
$$

$\mathrm{e}$

$$
T_{2} v=-\sqrt{\frac{\beta}{\beta^{\prime}}} v, \quad T_{2} w=\frac{1}{\beta^{\prime}}\left(\alpha^{\prime}+\alpha \sqrt{\frac{\beta}{\beta^{\prime}}}\right) v+w .
$$

2) As álgebras de $\operatorname{Bol} \mathcal{B}(\alpha, \beta, 0)$ e $\mathcal{B}\left(\alpha^{\prime}, \beta^{\prime}, 0\right)$, onde $\beta, \beta^{\prime} \in \mathbb{K}^{*}$, são isomorfas se, e somente se,

$$
\frac{\alpha^{2}}{\beta}=\frac{\alpha^{\prime 2}}{\beta^{\prime}}
$$

Demonstração: Pelo Corolário 2.1.7, $\mathcal{B}(\alpha, \beta, \gamma)$ é isomorfa a $\mathcal{B}\left(0,1, \gamma-\frac{\alpha^{2}}{\beta}\right)$ e $\mathcal{B}\left(\alpha^{\prime}, \beta^{\prime}, \gamma^{\prime}\right)$ é isomorfa a $\mathcal{B}\left(0,1, \gamma^{\prime}-\frac{\alpha^{\prime 2}}{\beta^{\prime}}\right)$. Do Corolário 2.1.8 temos que elas são isomorfas se, e somente se, $\gamma-\frac{\alpha^{2}}{\beta}=\gamma^{\prime}-\frac{\alpha^{\prime 2}}{\beta^{\prime}}$.

Do Corolário 2.1.5 temos que $a= \pm \sqrt{\frac{\beta}{\beta^{\prime}}}$. Se $a=\sqrt{\frac{\beta}{\beta^{\prime}}}$, então $c=\frac{1}{\beta^{\prime}}\left(\alpha^{\prime}-\alpha \sqrt{\frac{\beta}{\beta^{\prime}}}\right)$. Se $a=-\sqrt{\frac{\beta}{\beta^{\prime}}}$, então $c=\frac{1}{\beta^{\prime}}\left(\alpha^{\prime}+\alpha \sqrt{\frac{\beta}{\beta^{\prime}}}\right)$. Isto conclui a demonstração.

Corolário 2.1.10: As álgebras de $\operatorname{Bol} \mathcal{B}(0,0, \gamma)$ e $\mathcal{B}\left(0,0, \gamma^{\prime}\right)$, onde $\gamma, \gamma^{\prime} \in \mathbb{K}^{*}$, são isomorfas se, e somente se, $\gamma=\gamma^{\prime}$.

Lema 2.1.11: Seja $\alpha, \beta, \gamma \in \mathbb{K}$. Então temos o seguinte isomorfismo: $\mathcal{B}(\alpha, 0, \gamma) \simeq \mathcal{B}(1,0,0)$, se $\alpha \neq 0, \gamma \neq 0$.

Demonstração: Tome $a=\alpha \neq 0$ e $c=\frac{-\gamma}{2}$.

Teorema 2.1.12: Qualquer álgebra de Bol de dimensão 2 sobre $\mathbb{K}$ é isomorfa a uma das álgebras de Bol abaixo. Além disso, elas são duas a duas não isomorfas.

(I) $\mathcal{B}(0,0, \gamma)$, (II) $\mathcal{B}(1,0,0)$ e (III) $\mathcal{B}(0,1, \gamma)$. 
Demonstração: Do Corolário 2.1.5 temos que as álgebras de tipo (III ) não são isomorfas às do tipos (I) e (II). Suponha que $\mathcal{B}(1,0,0) \simeq \mathcal{B}(0,0, \gamma)$. Do Corolário 2.1.5 temos que $0=a(1-c \cdot 0)=a$ o que é um absurdo.

1) Se $\beta \neq 0$ temos, pelo Corolário 2.1.7, que $\mathcal{B}(\alpha, \beta, \gamma) \simeq \mathcal{B}\left(0,1, \gamma^{\prime}\right)$.

2) Se $\beta=0$ e $\alpha=0$ temos, pelo Corolário 2.1.10, uma família de álgebras de Bol não isomorfas entre si.

3) Se $\beta=0$ e $\alpha \neq 0$, pelo Lema 2.1.11 que $\mathcal{B}(\alpha, 0, \gamma) \simeq \mathcal{B}(1,0,0)$.

CASO II) Suponha que $a b=0$, para todo $a, b \in \mathcal{B}$. Notemos que neste caso a identidade 1.8 é sempre satisfeita, uma vez que todas as parcelas da identidade são nulas. Seja $\{v, w\}$ uma base de $\mathcal{B}$. Então temos que $v w=w v=v v=w w=0$.

Por fim, queremos definir em $\mathcal{B}$ uma estrutura de sistema triplo de Lie, isto é, definir $(\cdot ; \cdot, \cdot): \mathcal{B} \times \mathcal{B} \times \mathcal{B} \rightarrow \mathcal{B}$ de modo que as identidades (1.1), (1.2), (1.3) sejam satifeitas.

Definimos agora

$$
(v ; v, w)=\alpha v+\beta w=-(v ; w, v)
$$

e

$$
(w ; w, v)=\gamma v+\theta w=-(w ; v, w)
$$

onde $\alpha, \beta, \gamma, \theta$ são elementos do corpo $\mathbb{K}$. Nosso objetivo é determinar $\alpha, \beta, \gamma, \theta \in \mathbb{K}$ usando as identidades (1.3) e (1.8).

Agora, a identidade (1.3) vale com $a=x=y=v$ e $b=y=w$ se, e somente se,

$$
((v ; v, w) ; v, w)+(v ;(v ; v, w), w)+(v ; v,(w ; v, w))=((v ; v, w) ; v, w)
$$

ou seja, se, e somente se,

$$
(v ; \alpha v+\beta w, w)+(v ; v,-\gamma v-\theta w)=0
$$

isto é, se, e somente se,

$$
\alpha(v ; v, w)-\theta(v ; v, w)=0
$$

se, e somente se, 


$$
\alpha(\alpha v+\beta w)-\theta(\gamma v+\theta w)=0
$$

se, e somente se,

$$
\left\{\begin{array}{c}
(\alpha-\theta) \alpha=0 \\
(\alpha-\theta) \beta=0
\end{array},\right.
$$

e isto vale se, e somente se,

$$
\alpha=\theta \quad \text { ou } \quad \alpha=0 \quad \text { e } \quad \beta=0 .
$$

Então temos para o CASO II) os seguintes possibilidades:

$$
\text { i) }\left\{\begin{array}{l}
(v ; v, w)=\alpha v+\beta w \\
(w ; w, v)=\gamma v+\alpha w
\end{array} \quad i i\right)\left\{\begin{array}{l}
(v ; v, w)=0 \\
(w ; w, v)=\gamma v+\theta w
\end{array}\right. \text {. }
$$

Vimos anteriormente que o caso $(i)$ sempre é possível, para $\alpha, \beta, \gamma \in \mathbb{K}$. Veremos que o caso ( $i i)$ não fornece um sistema triplo de Lie. De fato, tomando $a=v, b=$ $w, x=w, y=v$ e $z=w$ na identidade 1.3 temos por um lado que $((x, y, z), a, b)=$ $\theta(w ; w, v)$ e por outro lado temos $((x, a, b), y, z)+(x,(y, a, b), z)+(x, y,(z, a, b))=$ $2 \theta(w ; w, v)$, que é possível se, e somente se, $\theta=0$.

Então temos o seguinte resultado.

Teorema 2.1.13: Sejam $\mathcal{B}$ um espaço vetorial de dimensão 2 sobre o corpo $\mathbb{K}$, $(\alpha, \beta, \gamma) \in \mathbb{K}^{3} \mathrm{e} \cdot$ uma operação bilinear em $\mathcal{B}$ tal que $v \cdot w=0$, para quaisquer $v, w \in \mathcal{B},(\cdot, \cdot, \cdot)$ uma operação trilinear

$$
(\cdot, \cdot, \cdot): \mathcal{B} \times \mathcal{B} \times \mathcal{B} \rightarrow \mathcal{B}
$$

tal que $(v, w, w)=(w, v, v)=0$ e $(v, v, w)=\alpha v+\beta w$ e $(w, w, v)=\gamma v+\alpha w$. Então $(\mathcal{B}, \cdot(\cdot, \cdot, \cdot))$ é uma álgebra de Bol sobre $\mathbb{K}$.

Agora vamos determinar, a menos de isomorfismo, todas as álgebras de Bol.

Teorema 2.1.14: Sejam $\mathcal{B}(\alpha, \beta, \gamma)$ e $\mathcal{B}\left(\alpha^{\prime}, \beta^{\prime}, \gamma^{\prime}\right)$ duas álgebras de Bol sobre $\mathbb{K}$. Então $\mathcal{B}(\alpha, \beta, \gamma)$ é isomorfa a $\mathcal{B}\left(\alpha^{\prime}, \beta^{\prime}, \gamma^{\prime}\right)$ se, e somente se, existem escalares $a, b, c, d \in$ 
$\mathbb{K}$ tal que

$$
\left\{\begin{array}{c}
a d-b c \neq 0 \\
\alpha a+\beta c=\alpha^{\prime}\left(a^{2} d-a b c\right)+\gamma^{\prime}\left(b^{2} c-b a d\right) \\
\alpha b+\beta d=\alpha^{\prime}\left(b^{2} c-b a d\right)+\gamma^{\prime}\left(a^{2} d-a b c\right) \\
\alpha c+\gamma a=\alpha^{\prime}\left(c^{2} b-c d a\right)+\gamma^{\prime}\left(d^{2} a-d c b\right) \\
\alpha d+\gamma b=\alpha^{\prime}\left(d^{2} a-d c b\right)+\gamma^{\prime}\left(c^{2} b-c d a\right)
\end{array}\right.
$$

ou equivalentemente

$$
\left\{\begin{array}{c}
a d-b c \neq 0 \\
\alpha a+\beta c-\left(a \alpha^{\prime}-b \gamma^{\prime}\right)(a d-b c)=0 \\
\alpha b+\beta d-\left(a \beta^{\prime}-b \alpha^{\prime}\right)(a d-b c)=0 \\
\alpha c+\gamma a-\left(d \gamma^{\prime}-c \alpha^{\prime}\right)(a d-b c)=0 \\
\alpha d+\gamma b-\left(d \alpha^{\prime}-c \beta^{\prime}\right)(a d-b c)=0
\end{array}\right.
$$

são satisfeitas.

Demonstração: Segue após um cálculo direto envolvendo a definição de isomorfismo de álgebra de Bol.

Lema 2.1.15: (1) As álgebras de $\operatorname{Bol} \mathcal{B}(1,0,0)$ e $\mathcal{B}(0,1,0)$ não são isomorfas.

(2) As álgebras de $\operatorname{Bol} \mathcal{B}(1,0,0)$ e $\mathcal{B}(0,0,1)$ não são isomorfas.

(3) As álgebras de $\operatorname{Bol} \mathcal{B}(0,1,0)$ e $\mathcal{B}(0,0,1)$ são isomorfas.

(4) Seja $\lambda \neq 0$. As álgebras de $\operatorname{Bol} \mathcal{B}(0,1, \gamma)$ e $\mathcal{B}(1,0,0)$ são isomorfas.

Demonstração: (1) Se as álgebras de Bol $\mathcal{B}(1,0,0)$ e $\mathcal{B}(0,1,0)$ fossem isomorfas, então pelo Teorema 2.1.14, existiriam escalares $a, b, c, d \in \mathbb{K}$ tal que valem as identidades do sistema (2.12). Mas da segunda igualdade segue que $a=0$ e, da terceira igualdade temos que $c=0$. Disto concluímos que $a d-b c=0$, o que é uma contradição. Portanto, as álgebras de $\operatorname{Bol} \mathcal{B}(1,0,0)$ e $\mathcal{B}(0,1,0)$ não são isomorfas.

(2) Se as álgebras de $\operatorname{Bol} \mathcal{B}(1,0,0)$ e $\mathcal{B}(0,0,1)$ fossem isomorfas, então pelo Teorema 2.1.14, existiriam escalares $a, b, c, d \in \mathbb{K}$ tal que valem as identidades do sistema (2.12). Da primeira igualdade temos $a=-b(a d-b c)$ e, da segunda igualdade temos 
$b=0$. Logo $a=0$ e, por conseguinte, $a d-b c=0$. Portanto as álgebras de Bol $\mathcal{B}(1,0,0)$ e $\mathcal{B}(0,0,1)$ não são isomorfas.

(3) Defina $T: \mathcal{B}(0,1,0) \rightarrow \mathcal{B}(0,0,1)$ da seguinte maneira: $T v=a x+y$ e $T w=x$. Note que $a d-b c=-1 \neq 0$.

(i) $\alpha a+\beta c-\left(a \alpha^{\prime}-b \gamma^{\prime}\right)(a d-b c)=0 . a+1-(0-1)(-1)=0$.

(ii) $\alpha b+\beta d-\left(a \beta^{\prime}-b \alpha^{\prime}\right)(a d-b c)=0 . b+\beta .0-(a .0-b .0)(-1)=0$.

(iii) $\alpha c+\gamma a-\left(d \gamma^{\prime}-c \alpha^{\prime}\right)(a d-b c)=0 . c+0 . a-\left(0 . \gamma^{\prime}-c .0\right)(-1)=0$.

(iv) $\alpha d+\gamma b-\left(d \alpha^{\prime}-c \beta^{\prime}\right)(a d-b c)=0 . d+0 . b-\left(0 . \alpha^{\prime}-c .0\right)(-1)=0$.

Portanto, pelo Teorema 2.1.14, segue que as álgebras de $\operatorname{Bol} \mathcal{B}(0,1,0)$ e $\mathcal{B}(0,0,1)$ são isomorfas.

(4) Defina $T: \mathcal{B}(0,1, \gamma) \rightarrow \mathcal{B}(1,0,0)$ do seguinte modo: $T v=\frac{1}{2} x-y$ e $T(w)=$ $\frac{\sqrt{-\gamma}}{2} x+\sqrt{-\gamma} y$. Note que $a d-b c=\sqrt{-\gamma} \neq 0$.

(i) $\alpha a+\beta c-\left(a \alpha^{\prime}-b \gamma^{\prime}\right)(a d-b c)=0 . a+\frac{\sqrt{-\gamma}}{2}-\left(\frac{1}{2}-b .0\right) \sqrt{-\gamma}=0$.

(ii) $\alpha b+\beta d-\left(a \beta^{\prime}-b \alpha^{\prime}\right)(a d-b c)=0 . b+\sqrt{-\gamma}-(a .0+1) \sqrt{-\gamma}=0$.

(iii) $\alpha c+\gamma a-\left(d \gamma^{\prime}-c \alpha^{\prime}\right)(a d-b c)=0 . c+\gamma \frac{1}{2}-\left(d .0-\frac{\sqrt{-\gamma}}{2}\right) \sqrt{-\gamma}=0$.

(iv) $\alpha d+\gamma b-\left(d \alpha^{\prime}-c \beta^{\prime}\right)(a d-b c)=0 . d+\gamma(-1)-(\sqrt{-\gamma}-c .0) \sqrt{-\gamma}=0$.

Portanto, as álgebras de $\operatorname{Bol} \mathcal{B}(0,1, \gamma)$ e $\mathcal{B}(1,0,0)$ são isomorfas.

Lema 2.1.16: Seja $(\alpha, \beta, \gamma) \in \mathbb{K}^{3}$. Então temos os seguintes isomorfismos de álgebras de Bol

(1) $\mathcal{B}(0,0, \gamma) \cong \mathcal{B}(0,0,1), \gamma \neq 0$.

(2) $\mathcal{B}(\alpha, 0, \gamma) \cong \mathcal{B}(1,0,0), \alpha \neq 0$ e $\gamma \neq 0$.

(3) $\mathcal{B}(\alpha, 0,0) \cong \mathcal{B}(1,0,0), \alpha \neq 0$.

(4) $\mathcal{B}(0, \beta, 0) \cong \mathcal{B}(0,1,0), \beta \neq 0$.

(5) $\mathcal{B}(\alpha, \beta, 0) \cong \mathcal{B}(0,1,0)$ se $\alpha \neq 0$ e $\gamma \neq 0$.

(6) $\mathcal{B}(\alpha, \beta, \gamma) \cong \mathcal{B}(1,0,0)$, se $\gamma \neq \frac{\alpha^{2}}{\beta}$ e $\mathcal{B}(\alpha, \beta, \gamma) \cong \mathcal{B}(0,1,0)$, se $\gamma=\frac{\alpha^{2}}{\beta}$.

Demonstração: (1) Suponha que $\gamma \neq 0$. Defina $T: \mathcal{B}(0,0, \gamma) \rightarrow \mathcal{B}(0,0,1)$ da 
seguinte forma: $T(v)=\sqrt{\gamma} x$ e $T(w)=\sqrt{\gamma} y$. Note que $a d-b c=\gamma \neq 0$.

(i) $\alpha a+\beta c-\left(a \alpha^{\prime}-b \gamma^{\prime}\right)(a d-b c)=0 . a+0 . c-\left(a .0-0 . \gamma^{\prime}\right) \gamma=0$.

(ii) $\alpha b+\beta d-\left(a \beta^{\prime}-b \alpha^{\prime}\right)(a d-b c)=0 . b+0 . d-\left(a .0-0 . \alpha^{\prime}\right) \gamma=0$.

(iii) $\alpha c+\gamma a-\left(d \gamma^{\prime}-c \alpha^{\prime}\right)(a d-b c)=0 . c+\gamma \sqrt{\gamma}-(\sqrt{\gamma}-c .0) \gamma=0$.

(iv) $\alpha d+\gamma b-\left(d \alpha^{\prime}-c \beta^{\prime}\right)(a d-b c)=0 . d+\gamma .0-(d .0-c .0)(a d-b c)=0$.

Portanto, as álgebras de $\operatorname{Bol} \mathcal{B}(0,0, \gamma) \cong \mathcal{B}(0,0,1)$, onde $\gamma \neq 0$.

(2) Defina $T: \mathcal{B}(\alpha, 0, \gamma) \rightarrow \mathcal{B}(1,0,0)$ da seguinte forma: $T(v)=\frac{2 \alpha}{\gamma} x$ e $T(w)=$ $-x+\frac{\gamma}{2} y$. Note que $a d-b c=\alpha \neq 0$.

(i) $\alpha a+\beta c-\left(a \alpha^{\prime}-b \gamma^{\prime}\right)(a d-b c)=\alpha \cdot \frac{2 \alpha}{\gamma}+0 . c-\left(\frac{2 \alpha}{\gamma}-0 \cdot \gamma^{\prime}\right) \alpha=0$.

(ii) $\alpha b+\beta d-\left(a \beta^{\prime}-b \alpha^{\prime}\right)(a d-b c)=\alpha .0+0 . d-\left(a .0-0 . \alpha^{\prime}\right) \alpha=0$.

(iii) $\alpha c+\gamma a-\left(d \gamma^{\prime}-c \alpha^{\prime}\right)(a d-b c)=-\alpha+\gamma \cdot \frac{2 \alpha}{\gamma}-(d .0-1) \alpha=0$.

(iv) $\alpha d+\gamma b-\left(d \alpha^{\prime}-c \beta^{\prime}\right)(a d-b c)=\alpha \cdot \frac{\gamma}{2}+\gamma \cdot 0-\left(\frac{\gamma}{2}-c .0\right) \alpha=0$.

Portanto, as álgebras de $\operatorname{Bol} \mathcal{B}(\alpha, 0, \gamma)$ e $\mathcal{B}(1,0,0)$ são isomorfas.

(3) Suponha $\alpha \neq 0$. Defina $T: \mathcal{B}(\alpha, 0,0) \rightarrow \mathcal{B}(1,0,0)$ da seguinte forma: $T(v)=$ $\sqrt{\alpha} x$ e $T(w)=\sqrt{\alpha} y$. Note que $a d-b c=\alpha \neq 0$.

(i) $\alpha a+\beta c-\left(a \alpha^{\prime}-b \gamma^{\prime}\right)(a d-b c)=\alpha \cdot \sqrt{\alpha}+0 . c-(\sqrt{\alpha}-b .0) \alpha=0$.

(ii) $\alpha b+\beta d-\left(a \beta^{\prime}-b \alpha^{\prime}\right)(a d-b c)=\alpha .0+0 . d-\left(a .0-0 . \alpha^{\prime}\right) \alpha=0$.

(iii) $\alpha c+\gamma a-\left(d \gamma^{\prime}-c \alpha^{\prime}\right)(a d-b c)=\alpha .0+0 . a-\left(d .0-0 . \alpha^{\prime}\right) \alpha=0$.

(iv) $\alpha d+\gamma b-\left(d \alpha^{\prime}-c \beta^{\prime}\right)(a d-b c)=\alpha \sqrt{\alpha}+0 . b-(\sqrt{\alpha}-c .0) \alpha=0$.

Portanto, as álgebras de $\operatorname{Bol} \mathcal{B}(\alpha, 0,0)$ e $\mathcal{B}(1,0,0)$ são isomorfas.

(4) Suponha $\beta \neq 0$. Defina $T: \mathcal{B}(0, \beta, 0) \rightarrow \mathcal{B}(0,1,0)$ da seguinte forma: $T(v)=$ $\sqrt{\beta} x$ e $T(w)=y$. Note que $a d-b c=\sqrt{\beta} \neq 0$.

(i) $\alpha a+\beta c-\left(a \alpha^{\prime}-b \gamma^{\prime}\right)(a d-b c)=0 . a+\beta .0-\left(a .0-0 . \gamma^{\prime}\right) \sqrt{\beta}=0$.

(ii) $\alpha b+\beta d-\left(a \beta^{\prime}-b \alpha^{\prime}\right)(a d-b c)=0 . b+\beta-\left(\sqrt{\beta}-0 . \alpha^{\prime}\right) \sqrt{\beta}=0$.

(iii) $\alpha c+\gamma a-\left(d \gamma^{\prime}-c \alpha^{\prime}\right)(a d-b c)=0 . c+0 . a-(d .0-c .0) \sqrt{\beta}=0$.

(iv) $\alpha d+\gamma b-\left(d \alpha^{\prime}-c \beta^{\prime}\right)(a d-b c)=0 . d+\gamma \cdot 0-\left(d .0-0 . \beta^{\prime}\right) \sqrt{\beta}=0$.

Portanto, as álgebras de $\operatorname{Bol} \mathcal{B}(0, \beta, 0)$ e $\mathcal{B}(0,1,0)$ são isomorfas.

(5) Suponha $(\alpha, \beta, \gamma) \neq(0,0,0)$. Defina $T: \mathcal{B}\left(0,1, \gamma-\frac{\alpha^{2}}{\beta}\right) \rightarrow \mathcal{B}(\alpha, \beta, \gamma)$ da seguinte forma: $T(v)=\frac{1}{\sqrt{\beta}} x$ e $T(w)=\frac{\alpha}{\beta} x+y$. Note que $a d-b c=\frac{1}{\sqrt{\beta}} \neq 0$.

(i) $\alpha a+\beta c-\left(a \alpha^{\prime}-b \gamma^{\prime}\right)(a d-b c)=0 . a+\frac{\alpha}{\beta}-\left(\frac{\alpha}{\sqrt{\beta}}-0 . \gamma^{\prime}\right) \frac{1}{\sqrt{\beta}}=0$.

(ii) $\alpha b+\beta d-\left(a \beta^{\prime}-b \alpha^{\prime}\right)(a d-b c)=0 . b+1-\left(\frac{\beta}{\sqrt{\beta}}-0 . \alpha^{\prime}\right) \frac{1}{\sqrt{\beta}}=0$. 
(iii) $\alpha c+\gamma a-\left(d \gamma^{\prime}-c \alpha^{\prime}\right)(a d-b c)=0 . c+\gamma-\frac{\alpha^{2}}{\beta} \cdot \frac{1}{\sqrt{\beta}}-\left(\gamma-\frac{\alpha}{\beta} \alpha\right) \frac{1}{\sqrt{\beta}}=0$.

(iv) $\alpha d+\gamma b-\left(d \alpha^{\prime}-c \beta^{\prime}\right)(a d-b c)=0 . d+\gamma \cdot 0-\left(\alpha-\frac{\alpha}{\beta} \cdot \beta\right) \frac{1}{\sqrt{\beta}}=0$.

Portanto, se $\gamma=\frac{\alpha^{2}}{\beta}$ temos que as álgebras de $\operatorname{Bol} \mathcal{B}(\alpha, \beta, \gamma)$ e $\mathcal{V}(0,1,0)$ são isomorfas. Se $\gamma \neq \frac{\alpha^{2}}{\beta}$, então temos que as álgebras de $\operatorname{Bol} \mathcal{B}(\alpha, \beta, \gamma)$ e $\mathcal{B}\left(0,1, \gamma-\frac{\alpha^{2}}{\beta}\right)$ são isomorfas, mas esta última é isomorfa a $\mathcal{B}(1,0,0)$ pelo nosso último lema.

Corolário 2.1.17: Seja $\mathbb{K}$ é um corpo algebricamente fechado de característica diferente de 2, existem a menos de isomorfismos apenas três algebras de Bol:

(I) $\mathcal{B}(0,0,0), \quad$ (II) $\mathcal{B}(1,0,0)$ e (III) $\mathcal{B}(0,1,0)$.

\section{2 Álgebra Envolvente Minimal}

O nosso objetivo agora é determinar as álgebras de Lie envolventes correspondentes a cada uma das álgebras de Bol classificadas nas seções anteriores. Primeiramente, determinaremos ipder $=\left\{D_{x, y} ; x, y \in \mathcal{B}\right\}$. Antes de começarmos, notemos os seguintes fatos resumidos no próximo lema.

Lema 2.2.1: (1) $D_{v, v}=D_{w, w}=0$;

(2) $D_{v, w}=-D_{w, v}$, onde $v$, w é uma base de $\mathcal{B}$ tal que $v \cdot w=v$;

(3) $D_{x, y}(z)=\left(a_{1} b_{2}-b_{1} a_{2}\right) D_{v, w}(z)$ para quaisquer $x, y, z \in \mathcal{B}, x=a_{1} v+a_{2} w \mathrm{e}$ $y=b_{1} v+b_{2} w$.

Demonstração: (1): De fato, dado $z \in \mathcal{B}$, temos pela identidade (1.4) que $D_{v, v}(z)=(z, v, v)=0=(z, w, w)=D_{w, w}(z)$.

(2): Seja $z=a v+b w \in \mathcal{B}$, onde $a, b \in \mathbb{K}$. Então $D_{v, w}(z)=(a v+b w, v, w)=$ $a(v, v, w)-b(w, w, v)$. Por outro lado, $D_{w, v}(z)=(a v+b w, w, v)=-a(v, v, w)+$ $b(w, w, v)$. Como $z$ é um elemento arbitrário de $\mathcal{B}$, segue que $D_{v, w}=-D_{w, v}$.

(3): Seja $x=a_{1} v+a_{2} w, y=b_{1} v+b_{2} w$. Então temos $D_{x, y}(z)=(z, x, y)=$ $\left(z, a_{1} v+a_{2} w, b_{1} v+b_{2} w\right)=a_{1} b_{2}(z, v, w)+b_{1} a_{2}(z, w, v)=a_{1} b_{2} D_{v, w}(z)+b_{1} a_{2} D_{w, v}(z)=$ $\left(a_{1} b_{2}-b_{1} a_{2}\right) D_{v, w}(z)$.

Da definição (1.4.25) e do Lema acima temos que o conjunto de todas as pseudo- 
derivações internas é

$$
\left\{D_{x, y} ; x, y \in \mathcal{B}\right\}=\left\{t D_{v, w} ; t \in \mathbb{K}\right\} .
$$

Isto mostra que as pseudoderivações internas é um espaço vetorial de dimensão 1 sobre o corpo $\mathbb{K}$. Além disso, $\operatorname{Ipder}(\mathcal{B})=\left\{\left(D_{x, y}, x \cdot y\right)\right\}$.

No que segue, denotaremos por $\operatorname{Lie} \mathcal{B}(\alpha, \beta, \gamma)$ a álgebra de Lie correspondente à álgebra de $\operatorname{Bol} \mathcal{B}(\alpha, \beta, \gamma)$.

Lema 2.2.2: Ponha $d=\left(D_{v, w}, v\right)$. Temos as seguintes álgebras de Lie.

(I) $\operatorname{Lie} \mathcal{B}(0,0, \gamma)$ com base $\{v, w, d\}$ e produto dado por:

$$
[v, w]=-d,[v, d]=0,[w, d]=\gamma v
$$

Se $\gamma=0$, então $\operatorname{Lie} \mathcal{B}(0,0,0)$ é a álgebra de Lie abeliana de dimensão 2 .

(II) $\operatorname{Lie} \mathcal{B}(1,0,0)$ com base $\{v, w, d\}$ e produto dado por:

$$
[v, w]=-d,[v, d]=-v,[w, d]=w
$$

(III) $\operatorname{Lie} \mathcal{B}(0,1,0)$ com base $\{v, w, d\}$ e produto dado por:

$$
[v, w]=-d,[v, d]=-w,[w, d]=0
$$

(IV) Lie $\mathcal{B}(0,1, \gamma), \gamma \neq 0$ com base $\{v, w, d\}$ e produto dado por:

$$
[v, w]=-d,[v, d]=-w,[w, d]=\gamma v
$$

Demonstração: Vamos aplicar a Definição (1.4.29) para determinar cada uma das álgebras de Lie dadas no enunciado do Lema.

$(\mathrm{I}): \mathcal{B}(0,0, \gamma)$ tem a seguinte multiplicação: $(v, v, w)=0$ e $(w, w, v)=\gamma v$. Segue que $[v, w]=-\left(D_{v, w}, v\right)=-d,[v, d]=-d(v)=-(v, v, w)=0,[w, d]=-d(w)=$ $-(w, v, w)=(w, w, v)=\gamma v$.

(II): $\mathcal{B}(1,0,0)$ tem a seguinte multiplicação: $(v, v, w)=v$ e $(w, w, v)=w$. Segue que $[v, w]=-\left(D_{v, w}, v\right)=-d,[v, d]=-d(v)=-(v, v, w)=-v,[w, d]=-d(w)=$ $-(w, v, w)=(w, w, v)=w$.

(III): $\mathcal{B}(0,1,0)$ tem a seguinte multiplicação: $(v, v, w)=w$ e $(w, w, v)=0$. Segue 
que $[v, w]=-\left(D_{v, w}, v\right)=-d,[v, d]=-d(v)=-(v, v, w)=-v,[w, d]=-d(w)=$ $-(w, v, w)=(w, w, v)=0$.

(III): $\mathcal{B}(0,1, \gamma), \gamma \neq 0$ tem a seguinte multiplicação: $(v, v, w)=w$ e $(w, w, v)=\gamma v$. Segue que $[v, w]=-\left(D_{v, w}, v\right)=-d,[v, d]=-d(v)=-(v, v, w)=-w,[w, d]=$ $-d(w)=-(w, v, w)=(w, w, v)=\gamma v$.

No próximo lema, mostraremos que existem álgebras de Bol não isomorfas cujas álgebras de Lie correspondentes são isomorfas.

Lema 2.2.3: Temos os seguintes isomorfismos de álgebras de Lie.

(1) $\operatorname{Lie} \mathcal{B}(0,0, \gamma) \simeq \operatorname{Lie} \mathcal{B}(0,1,0)$.

(2) $\operatorname{Lie} \mathcal{B}(1,0,0) \simeq \operatorname{Lie} \mathcal{B}(0,1, \gamma)$.

(3) As álgebras $\operatorname{Lie} \mathcal{B}(1,0,0)$ e $\operatorname{Lie} \mathcal{B}(0,1,0)$ não são isomorfas.

Demonstração: Lembremos que existe uma única álgebra de Lie simples de dimensão 3 sobre $\mathbb{K}$, onde $\mathbb{K}$ é um corpo algebricamente fechado.

(1) Seja $\{v, w, d\}$ uma base de $\operatorname{LieB}(0,0, \gamma)$ satisfazendo a multiplicação definida acima e $\left\{v^{\prime}, w^{\prime}, d^{\prime}\right\}$ uma base de $\operatorname{Lie} \mathcal{B}(0,1,0)$ satisfazendo a mesma regra de multiplicação. Então a aplicação $T: \operatorname{Lie} \mathcal{B}(0,0, \gamma) \rightarrow \operatorname{Lie} \mathcal{B}(0,1,0)$, definida por $T v=$ $d^{\prime}, T w=\sqrt{\gamma} v^{\prime}, T d=-\sqrt{\gamma} w^{\prime}$ é um isomorfismo de álgebras de Lie. De fato, $T(-d)=-T(d)=-\left(-\sqrt{\gamma} w^{\prime}\right)=\sqrt{\gamma} w^{\prime}=\sqrt{\gamma}\left[d^{\prime}, v^{\prime}\right]=\left[d^{\prime}, \sqrt{\gamma} v^{\prime}\right]=[T v, T w] ; T(0)=$ $0=-\sqrt{\gamma}\left[d^{\prime}, w^{\prime}\right]=\left[d^{\prime},-\sqrt{\gamma} w^{\prime}\right]=[T v, T d] ; T(\gamma v)=\gamma T(v)=\gamma d^{\prime}=-\gamma(-d)=$ $-\gamma\left[v^{\prime}, w^{\prime}\right]=\left[\sqrt{\gamma} v^{\prime},-\sqrt{\gamma} w^{\prime}\right]=[T w, T d]$.

(2) Pela observação no início, temos que $\operatorname{Lie} \mathcal{B}(1,0,0) \simeq \operatorname{Lie\mathcal {B}}(0,1, \gamma)$.

(3) As álgebras de Lie no enunciado não são isomorfas pois uma é simples e a outra não é simples.

\section{3 Álgebras de Bol de dimensão 2 sobre um corpo que não é algebricamente fechado}

Vamos considerar o caso quando não necessariamente todo elemento do corpo é igual a um quadrado, ou seja, a identidade $\beta=a^{2} \beta^{\prime}$ não necessariamente é satisfeita em $\mathbb{K}$. 
Neste caso, consideramos o seguinte grupo quociente $\mathbb{K}^{*} /\left(\mathbb{K}^{*}\right)^{2}$, onde $\left(\mathbb{K}^{*}\right)^{2}=\left\{a^{2}\right.$ : $a \in \mathbb{K}\}$. Denotemos por $[\alpha]=\left\{\alpha^{\prime} \in \mathbb{K} \mid\right.$ existe $a \in \mathbb{K}$ tal que $\left.\alpha=a^{2} \alpha^{\prime}\right\}$. Então temos que $\mathcal{B}([\alpha],[\beta],[\gamma]) \simeq \mathcal{B}\left(\left[\alpha^{\prime}\right],\left[\beta^{\prime}\right],\left[\gamma^{\prime}\right]\right)$ se, e somente se, $\alpha \approx \alpha^{\prime}, \beta \approx \beta^{\prime}$ e $\gamma \approx \gamma^{\prime}$.

Procedendo como na seção anterior obtemos resultados análogos aos daquela seção. Por exemplo, temos o seguinte:

Teorema 2.3.1: $\quad$ Duas álgebras de $\operatorname{Bol} \mathcal{B}(\alpha, \beta, \gamma)$ e $\mathcal{B}\left(\alpha^{\prime}, \beta^{\prime}, \gamma^{\prime}\right)$ são isomorfas se, e somente se, as relações

(1) $\beta \approx \beta^{\prime}$,

(2) $\alpha=a\left(\alpha^{\prime}-c \beta^{\prime}\right)$,

(3) $\gamma=\gamma^{\prime}-c\left(2 \alpha^{\prime}-c \beta^{\prime}\right)$,

são satisfeitas.

Teorema 2.3.2: Qualquer álgebra de Bol, de dimensão 2, sobre um corpo de característica 0 , que não é algebricamente fechado, encaixa-se num dos casos abaixo.

(I) $\mathcal{B}(0,0,[\gamma])$

(II) $\mathcal{B}(1,0,0)$.

(III) $\mathcal{B}(0,[\beta], 0)$.

(IV) $\mathcal{B}(0,[\beta],[\gamma])$.

Como antes, temos o seguinte lema:

Lema 2.3.3: Ponha $d=\left(D_{v, w}, 0\right)$. Temos as seguintes álgebras de Lie:

(I) $\operatorname{Lie}(0,0, \gamma)$ com base $\{v, w, d\}$ e produto dado por

$$
[v, w]=-d,[v, d]=0,[w, d]=\gamma v
$$

(II) Lie $(1,0,0)$ com base $\{v, w, d\}$ e produto dado por

$$
[v, w]=-d,[v, d]=-v[w, d]=w
$$

(III) Lie $(0, \beta, 0)$ com base $\{v, w, d\}$ e produto dado por

$$
[v, w]=-d,[v, d]=-\beta w,[w, d]=0
$$


(IV) Lie $(0, \beta, \gamma)$ com base $\{v, w, d\}$ e produto dado por

$$
[v, w]=-d,[v, d]=-\beta w,[w, d]=\gamma v
$$

Demonstração: A prova é exatamente a que foi feita no Lema (2.2.2). 


\section{Capítulo 3}

\section{Loops de Bol algébricos e analíticos de dimensão 2}

Neste capítulo provaremos que todo loop de Bol analítico (algébrico) de dimensão 2 sobre um corpo de característica 0 é um grupo.

\subsection{Loops de Bol algébricos e analíticos de dimensão 2 sobre um corpo algebricamente fechado}

Nosso primeiro passo é determinar os grupos algébricos correspondentes a cada uma das álgebras de Lie sobre um corpo algebricamente fechado que foram determinadas no Capítulo anterior.

(I) $\mathrm{O}$ grupo algébrico da álgebra de Lie $\operatorname{Lie} \mathfrak{B}(0,0, \gamma)$.

Não há nada para fazer no caso $\gamma=0$.

Lema 3.1.1: Seja $\mathfrak{L}$ uma álgebra de Lie de dimensão 3 sobre o corpo $\mathbb{K}$ com base $\{a, b, c\}$ satisfazendo a seguinte regra de multiplicação: $[a, b]=b,[a, c]=-c,[b, c]=$ 0 . Então $\mathfrak{L}$ pode ser mergulhada na álgebra de Lie das matrizes $3 \times 3, G L(3, \mathbb{K})$, via representação adjunta. 
Então $a \mapsto\left(\begin{array}{ccc}0 & 0 & 0 \\ 0 & -1 & 0 \\ 0 & 0 & 1\end{array}\right), b \mapsto\left(\begin{array}{ccc}0 & 1 & 0 \\ 0 & 0 & 0 \\ 0 & 0 & 0\end{array}\right), c \mapsto\left(\begin{array}{ccc}0 & 0 & -1 \\ 0 & 0 & 0 \\ 0 & 0 & 0\end{array}\right)$

Demonstração: Basta usar a definição de representação adjunta e as regras de multiplicação dos elementos da base.

Lema 3.1.2: $\quad$ Considere a álgebra de Lie $\operatorname{Lie} \mathcal{B}(0,0, \gamma), \gamma \neq 0$. Então existe uma base $\{a, b, c\}$ tal que $[a, b]=b,[a, c]=-c,[b, c]=0$, isto é, Lie $\mathcal{B}(0,0, \gamma)=\langle a>\oplus N$, onde $N$ é um ideal nilpotente gerado por $\{b, c\}$.

Demonstração: Ponha $a=\frac{1}{\sqrt{\gamma}} w, b=\frac{\sqrt{\gamma}}{2} v+\frac{1}{2} d$ e $c=\frac{\sqrt{\gamma}}{2} v-\frac{1}{2} d$. Então $\{a, b, c\}$ é uma base com a propriedade requerida.

De acordo com o Lema 3.1.1,

$$
\operatorname{Lie} \mathcal{B}(0,0, \gamma)=\left\{\left(\begin{array}{ccc}
0 & x & y \\
0 & -t & 0 \\
0 & 0 & t
\end{array}\right) ; x, y, t \in \mathbb{K}\right\}
$$

O grupo algébrico correspondente a álgebra de Lie acima é:

$$
G \mathcal{B}(0,0, \gamma)=\left\{\left(\begin{array}{ccc}
1 & x & y \\
0 & t^{-1} & 0 \\
0 & 0 & t
\end{array}\right) ; x, y, \in \mathbb{K}, t \in \mathbb{K}^{*}\right\}
$$

Na base $\{a, b, c\}$ do Lema 3.1.2 temos que $v=\frac{b+c}{\sqrt{\gamma}}, w=\sqrt{\gamma} a$ e $d=b-c$. Estes vetores correspondem as seguintes matrizes:

$$
w \mapsto\left(\begin{array}{ccc}
0 & 0 & 0 \\
0 & -\sqrt{\gamma} & 0 \\
0 & 0 & \sqrt{\gamma}
\end{array}\right), v \rightarrow\left(\begin{array}{ccc}
0 & \frac{1}{\sqrt{\gamma}} & -\frac{1}{\sqrt{\gamma}} \\
0 & 0 & 0 \\
0 & 0 & 0
\end{array}\right), d \mapsto\left(\begin{array}{ccc}
0 & 1 & 1 \\
0 & 0 & 0 \\
0 & 0 & 0
\end{array}\right)
$$


Considere agora o seguinte subgrupo de $G$ :

$$
H=\{\exp (t d) \mid t \in \mathbb{K}\}=\left\{\left(\begin{array}{ccc}
1 & x & x \\
0 & 1 & 0 \\
0 & 0 & 1
\end{array}\right) ; x \in \mathbb{K}\right\} .
$$

Considere também a seguinte subvariedade de dimensão 2 de $G$ :

$$
B=\{\exp (t w) \exp (s v) \exp (t w) \mid s, t \in \mathbb{K}\}=\left\{\left(\begin{array}{ccc}
1 & x t^{-1} & -x t \\
0 & t^{-2} & 0 \\
0 & 0 & t^{2}
\end{array}\right) \mid t \in \mathbb{K}^{*}, x \in \mathbb{K}\right\} .
$$

Lema 3.1.3: A subvariedade $B$ construída acima é um subgrupo torcido do grupo $G$. Além disso, $B$ não é um loop de Bol algébrico global.

Demonstração: 1) Pondo $x=0$ e $t=1$ vemos que $E \in B$.

2) Se $\alpha=\left(\begin{array}{ccc}1 & x t^{-1} & -x t \\ 0 & t^{-2} & 0 \\ 0 & 0 & t^{2}\end{array}\right) \in B$, então $\alpha^{-1}=\left(\begin{array}{ccc}1 & -x t & x t^{-1} \\ 0 & t^{2} & 0 \\ 0 & 0 & t^{-2}\end{array}\right) \in B$.

3) Seja $\alpha=\left(\begin{array}{ccc}1 & x t^{-1} & -x t \\ 0 & t^{-2} & 0 \\ 0 & 0 & t^{2}\end{array}\right)$ e $\beta=\left(\begin{array}{ccc}1 & y s^{-1} & -y s \\ 0 & s^{-2} & 0 \\ 0 & 0 & s^{2}\end{array}\right)$ dois elementos quaisquer de $B$. Então

$$
\alpha \beta \alpha=\left(\begin{array}{ccc}
1 & x t^{-1}+y s^{-1}+x t^{-3} s^{-2} & -x t-y s t^{2}-x s^{2} t^{3} \\
0 & \left(t^{2} s\right)^{-2} & 0 \\
0 & 0 & \left(t^{2} s\right)^{2}
\end{array}\right)=\left(\begin{array}{ccc}
1 & z r^{-1} & -z r \\
0 & r^{-2} & 0 \\
0 & 0 & r^{2}
\end{array}\right),
$$

onde $r=t^{2} s$ e $z=x t s+y+x t^{-1} s^{-1}$. Portanto $B$ é, por definição, um subgrupo torcido de $G$. Notemos que $B H=\left\{\left(\begin{array}{ccc}1 & a+x t^{-1} & a-x t \\ 0 & t^{-2} & 0 \\ 0 & 0 & t^{2}\end{array}\right) ; a, x \in \mathbb{K}\right.$ e $\left.t \in \mathbb{K}^{*}\right\}$. O elemento $\left(\begin{array}{lll}1 & 1 & 1 \\ 0 & 1 & 0 \\ 0 & 0 & 1\end{array}\right)$ pertence a $G$ mas não pertence a $B H$. Portanto, $B$ não é um loop de Bol algébrico global. 
(II) O grupo algébrico da álgebra de Lie Ließ $\mathcal{B}(0,1,0)$

Lema 3.1.4: A álgebra de Lie $\operatorname{Lie} \mathcal{B}(0,1,0)$ é isomorfa a álgebra de Lie

$$
\operatorname{Lie\mathcal {B}}(0,1,0)=\left\{\left(\begin{array}{ccc}
0 & -x+y & -x-y \\
0 & -z & 0 \\
0 & 0 & z
\end{array}\right) \mid t, x, y \in \mathbb{K}\right\} \text {. }
$$

Demonstração: Sabemos do Lema 2.2.3 que $\operatorname{Lie} \mathcal{B}(0,0,1)$ é isomorfa a $\operatorname{Lie} \mathcal{B}(0,1,0)$ e o isomorfismo é dado por $T v^{\prime}=d, T w^{\prime}=v$ e $T d^{\prime}=-w$, onde $v^{\prime}, w^{\prime}, d^{\prime}$ é uma base de $\operatorname{Lie} \mathcal{B}(0,0,1)$ tal que $\left[v^{\prime}, w^{\prime}\right]=-d^{\prime},\left[v^{\prime}, d^{\prime}\right]=0,\left[w^{\prime}, d^{\prime}\right]=v^{\prime}$. Da observação feita depois do Lema 3.1.2, temos que $v, w$ e $d$ representam-se matricialmente do seguinte modo:

$$
v=\left(\begin{array}{ccc}
0 & 0 & 0 \\
0 & -1 & 0 \\
0 & 0 & 1
\end{array}\right) w=\left(\begin{array}{ccc}
0 & -1 & -1 \\
0 & 0 & 0 \\
0 & 0 & 0
\end{array}\right) \text { e } d=\left(\begin{array}{ccc}
0 & 1 & -1 \\
0 & 0 & 0 \\
0 & 0 & 0
\end{array}\right)
$$

Tomando uma combinação linear destes elementos obtemos a álgebra de Lie do enunciado acima.

Lema 3.1.5: O subconjunto

$$
G=\left\{\left(\begin{array}{ccc}
1 & -x+y & -x-y \\
0 & t^{-1} & 0 \\
0 & 0 & t
\end{array}\right) \mid x, y \in \mathbb{K}, t \in \mathbb{K}^{*}\right\}
$$

das matrizes $3 \times 3$ é um grupo algébrico cuja álgebra de Lie é $\operatorname{Lie} \mathcal{B}(0,1,0)$.

\section{Demonstração:}

Note que temos

$$
\exp (s d)=\left(\begin{array}{ccc}
1 & s & -s \\
0 & 1 & 0 \\
0 & 0 & 1
\end{array}\right), \exp \left(t^{\prime} v\right)=\left(\begin{array}{ccc}
1 & 0 & 0 \\
0 & t^{-1} & 0 \\
0 & 0 & t
\end{array}\right), \exp (x w)=\left(\begin{array}{ccc}
1 & -x & -x \\
0 & 1 & 0 \\
0 & 0 & 1
\end{array}\right) .
$$


Portanto $H=\{\exp (s d) \mid s \in \mathbb{K}\}=\left\{\left(\begin{array}{ccc}1 & s & -s \\ 0 & 1 & 0 \\ 0 & 0 & 1\end{array}\right) \mid s \in \mathbb{K}\right\}$. Note que $H$ é um subgrupo de $G$ e

$B=\left\{\operatorname{esxp}\left(t^{\prime} v\right) \exp (x w) \exp \left(t^{\prime} v\right) \mid t^{\prime}, x \in \mathbb{K}\right\}=\left\{\left(\begin{array}{ccc}1 & -x t^{-1} & -x t \\ 0 & t^{-2} & 0 \\ 0 & 0 & t^{2}\end{array}\right) \mid x \in \mathbb{K}, t \in \mathbb{K}^{*}\right\}$

é uma subvariedade afim de dimensão 2 .

Lema 3.1.6: A subvariedade $B$, construída acima é um subgrupo torcido de $G$, que não é um loop de Bol algébrico global.

Demonstração: Pondo $x=0$ e $t=1$ temos que $E \in B$. Seja $\alpha=\left(\begin{array}{ccc}1 & -x t^{-1} & -x t \\ 0 & t^{-2} & 0 \\ 0 & 0 & t^{2}\end{array}\right)$ um elemento qualquer de $B$. Como $\alpha^{-1}=\left(\begin{array}{ccc}1 & x t & x t^{-1} \\ 0 & t^{2} & 0 \\ 0 & 0 & t^{-2}\end{array}\right)$, concluímos que $\alpha^{-1} \in$ B. Finalmente sejam $\alpha=\left(\begin{array}{ccc}1 & -x t^{-1} & -x t \\ 0 & t^{-2} & 0 \\ 0 & 0 & t^{2}\end{array}\right)$ e $\beta=\left(\begin{array}{ccc}1 & -y s^{-1} & -y s \\ 0 & s^{-2} & 0 \\ 0 & 0 & s^{2}\end{array}\right)$ dois elementos arbitrários de $B$. Como $\alpha \beta \alpha$ é igual a

$$
\left(\begin{array}{ccc}
1 & -x t^{-1}-y s^{-1} t^{-2}-x s^{-2} t^{-3} & -x t-y s t^{2}-x s^{2} t^{3} \\
0 & \left(t^{2} s\right)^{-2} & 0 \\
0 & 0 & \left(t^{2} s\right)^{2}
\end{array}\right)=\left(\begin{array}{ccc}
1 & -z r^{-1} & -z r \\
0 & r^{-1} & 0 \\
0 & 0 & r
\end{array}\right)
$$

que por sua vez é um elemento de $B$, onde $r=s t^{2}$ e $z=x s^{-1} t^{-1}+y+x s t$. Portanto $B$ é, por definição, um subgrupo torcido de $G$. 
Agora vamos determinar $B H$. Note que

$$
B H=\left\{\left(\begin{array}{ccc}
1 & s-x t^{-1} & -s-x t \\
0 & t^{-2} & 0 \\
0 & 0 & t^{2}
\end{array}\right) \mid x, s \in \mathbb{K}, t \in \mathbb{K}^{*}\right\}
$$

Mas o elemento $\alpha=\left(\begin{array}{ccc}1 & 0 & 0 \\ 0 & -1 & 0 \\ 0 & 0 & -1\end{array}\right)$ pertence a $G$ mas não pertence a $B H$. Portanto, $B$ não é um loop de Bol algébrico global.

(III) O grupo algébrico da álgebra de Lie $\operatorname{Lie} \mathcal{B}(0,1, \gamma), \gamma \neq 0$.

Lembremos que $\operatorname{Lie} \mathcal{B}(0,1, \gamma)$, onde $\gamma \neq 0$ tem base $\{v, w, d\}$ e multiplicação dada por: $[v, w]=-d,[v, d]=-w,[w, d]=\gamma v$. Como LieB $(0,1, \gamma)$ é simples, então ela é isomorfa a $\mathfrak{s l}(2, \mathbb{K})$ e escrevendo

$$
v=X+1 / 4 Y, \quad w=-\sqrt{-\gamma} X+\frac{\sqrt{-\gamma}}{4} Y, \quad d=\frac{-\sqrt{-\gamma}}{2} H,
$$

onde $\{X, H, Y\}$ é base de $\mathfrak{s l}(2, \mathbb{K})$ temos:

$$
v=\left(\begin{array}{cc}
0 & 1 \\
1 / 4 & 0
\end{array}\right), w=\left(\begin{array}{cc}
0 & \sqrt{-\gamma} \\
\frac{\sqrt{-\gamma}}{4} & 0
\end{array}\right) \text { e } d=\left(\begin{array}{cc}
-\frac{\sqrt{-\gamma}}{2} & 0 \\
0 & \frac{\sqrt{-\gamma}}{2}
\end{array}\right) .
$$

Seja $H=\left\{e^{r d} \mid r \in \mathbb{K}\right\}=\left\{\left(\begin{array}{cc}t^{-1} & 0 \\ 0 & t\end{array}\right) \mid t \in \mathbb{K}^{*}\right\}$ e note que

$$
v=\left(\begin{array}{cc}
\frac{1}{2} & \frac{1}{2} \\
-\frac{1}{4} & \frac{1}{4}
\end{array}\right)\left(\begin{array}{cc}
-\frac{1}{2} & 0 \\
0 & \frac{1}{2}
\end{array}\right)\left(\begin{array}{cc}
1 & -2 \\
1 & 2
\end{array}\right)
$$

$\mathrm{e}$

$$
w=\left(\begin{array}{cc}
\frac{1}{2} & -\frac{i}{2} \\
-\frac{i}{4} & \frac{1}{4}
\end{array}\right)\left(\begin{array}{cc}
-\frac{\sqrt{\gamma}}{2} & 0 \\
0 & \frac{\sqrt{\gamma}}{2}
\end{array}\right)\left(\begin{array}{cc}
1 & 2 i \\
i & 2
\end{array}\right)
$$

Então

$$
e^{t^{\prime} v}=\left(\begin{array}{cc}
\frac{1}{2}\left(t+t^{-1}\right) & t-t^{-1} \\
\frac{1}{4}\left(t-t^{-1}\right) & \frac{1}{2}\left(t+t^{-1}\right)
\end{array}\right)=\left(\begin{array}{cc}
2 u & 4 v \\
v & 2 u
\end{array}\right)
$$


e

$$
e^{s^{\prime} w}=\left(\begin{array}{cc}
\frac{1}{2}\left(s+s^{-1}\right) & -i\left(s-s^{-1}\right) \\
\frac{i}{4}\left(s-s^{-1}\right) & \frac{1}{2}\left(s+s^{-1}\right)
\end{array}\right)=\left(\begin{array}{cc}
2 u^{\prime} & -4 v^{\prime} \\
v^{\prime} & 2 u^{\prime}
\end{array}\right),
$$

onde $u=\frac{1}{4}\left(t+t^{-1}\right), v=\frac{1}{4}\left(t-t^{-1}\right), u^{\prime}=\frac{1}{4}\left(s+s^{-1}\right), v^{\prime}=\frac{i}{4}\left(s+s^{-1}\right)$, para $s, t \in \mathbb{K} *$. Seja

$$
\begin{aligned}
B & =\left\{\left(\begin{array}{cc}
2 u & 4 v \\
v & 2 u
\end{array}\right)\left(\begin{array}{cc}
2 u^{\prime} & -4 v^{\prime} \\
v^{\prime} & 2 u^{\prime}
\end{array}\right)\left(\begin{array}{cc}
2 u & 4 v \\
v & 2 u
\end{array}\right) \mid u, u^{\prime}, v, v^{\prime} \in \mathbb{K}\right\} \\
& =\left\{\left(\begin{array}{cc}
8 u^{2} u^{\prime}+8 v^{2} v^{\prime} & -16 u^{2} v+32 u u^{\prime} v+16 v^{2} v^{\prime} \\
4 u^{2} v^{\prime}+8 u u^{\prime} v-4 v^{2} v^{\prime} & 8 u^{2} u^{\prime}+8 v^{2} v^{\prime}
\end{array}\right) \mid u, u^{\prime}, v, v^{\prime} \in \mathbb{K}\right\} \\
& =\left\{\left(\begin{array}{ll}
a & b \\
c & a
\end{array}\right) \mid a^{2}=b c+1 \mathrm{e} c, d \in \mathbb{K}\right\} .
\end{aligned}
$$

Acima tomemos $a=8 u^{2} u^{\prime}+8 v^{2} v^{\prime}, b=-16 u^{2} v+32 u u^{\prime} v+16 v^{2} v^{\prime}$ e $4 u^{2} v^{\prime}+8 u u^{\prime} v-4 v^{2} v^{\prime}$.

Lema 3.1.7: A variedade afim $B$ é um subgrupo torcido de $G$ mas não é um loop de Bol algébrico global.

Demonstração: A prova é inteiramente análoga a feita no próximo Lema.

(IV) O grupo algébrico da álgebra de Lie $\operatorname{Lie} \mathcal{B}(1,0,0)$.

A álgebra de Lie $\operatorname{Lie} \mathcal{B}(1,0,0)$ é simples e, portanto, isomorfa a álgebra de Lie $\operatorname{sl}(2, \mathbb{K})$. Vamos escrever cada elemento da base de $\operatorname{Lie} \mathcal{B}(1,0,0)$ como combinação linear da base de $\operatorname{sl}(2, \mathbb{K})$. Sabemos que $\operatorname{sl}(2, \mathbb{K})$ tem base $\{X, Y, H\}$ tal que

$$
[H, X]=2 X,[H, Y]=-2 Y[X, Y]=H
$$

e estes elementos correspondem os seguintes elementos de $g l(2, \mathbb{K})$;

$$
X \mapsto\left(\begin{array}{cc}
0 & 1 \\
0 & 0
\end{array}\right), Y \mapsto\left(\begin{array}{cc}
0 & 0 \\
1 & 0
\end{array}\right), H \mapsto\left(\begin{array}{cc}
1 & 0 \\
0 & -1
\end{array}\right)
$$

Como $\{v, w, d\}$ é uma base de $\operatorname{Lie\mathcal {B}}(1,0,0)$ com a regra de multiplicação dada por $[v, w]=-d,[v, d]=-v[w, d]=w$, temos que $v=\frac{-1}{2} X, w=Y$ e $d=\frac{1}{2} H$ que na 
forma matricial escreve-se como:

$$
v=\left(\begin{array}{cc}
0 & \frac{-1}{2} \\
0 & 0
\end{array}\right), w=\left(\begin{array}{cc}
0 & 0 \\
1 & 0
\end{array}\right), d=\left(\begin{array}{cc}
\frac{1}{2} & 0 \\
0 & \frac{-1}{2}
\end{array}\right) .
$$

O grupo algébrico correspondente a $s l(2, \mathbb{K})$ é o grupo das matrizes $2 \times 2$ cujo determinante é igual a 1. Agora temos que

$$
H=\{\exp (s d) \mid s \in \mathbb{K}\}=\left\{\left(\begin{array}{cc}
t & 0 \\
0 & t^{-1}
\end{array}\right) \mid t \in \mathbb{K}^{*}\right\} .
$$

Vamos considerar o subgrupo torcido $B=\left\{\exp (t w) \exp (s v) \exp (t w) \mid s, t \in \mathbb{K}^{*}\right\}$. Inicialmente notemos que $v$ e $w$ são matrizes nilpotentes de ordem 2. Vamos deter$\operatorname{minar} \exp (t w)$.

$$
\exp (t w)=\left(\begin{array}{ll}
1 & 0 \\
0 & 1
\end{array}\right)+t\left(\begin{array}{ll}
0 & 0 \\
1 & 0
\end{array}\right)=\left(\begin{array}{ll}
1 & 0 \\
t & 1
\end{array}\right) .
$$

Da mesma forma temos

$$
\exp (s v)=\left(\begin{array}{cc}
1 & 0 \\
0 & 1
\end{array}\right)+s\left(\begin{array}{cc}
0 & \frac{-1}{2} \\
0 & 0
\end{array}\right)=\left(\begin{array}{cc}
1 & \frac{-s}{2} \\
0 & 1
\end{array}\right)
$$

Então

$$
\begin{gathered}
B=\{\exp (t w) \exp (s v) \exp (t w) \mid s, t \in \mathbb{K}\}=\left\{\left(\begin{array}{ll}
1 & 0 \\
t & 1
\end{array}\right)\left(\begin{array}{ll}
1 & \frac{-s}{2} \\
0 & 1
\end{array}\right)\left(\begin{array}{ll}
1 & 0 \\
t & 1
\end{array}\right)\right\}= \\
=\left\{\left(\begin{array}{cc}
\frac{-t s}{2}+1 & \frac{-s}{2} \\
\frac{-t^{2} s}{2}+2 t & \frac{-t s}{2}+1
\end{array}\right) \mid t, s \in \mathbb{K}\right\} . \\
\text { Pondo } a=\frac{-t s}{2}+1 \text { e } b=\frac{-t^{2} s}{2}+2 t \text { temos que } \\
B=\left\{\left(\begin{array}{cc}
a & s \\
b & a
\end{array}\right) \mid s b=a^{2}-1, t, s \in \mathbb{K}\right\} .
\end{gathered}
$$

Lema 3.1.8: A subvariedade $B$ construída acima é um subgrupo torcido de $G$, mas não é um loop de Bol algébrico global. 
Demonstração: 1) Ponha $b=s=0$ e $a=1$. Logo $E \in B$.

2) Se $x=\left(\begin{array}{ll}a & s \\ b & a\end{array}\right) \in B$ então $x^{-1}=\left(\begin{array}{cc}a & -s \\ -b & a\end{array}\right)$ é um elemento de $B$.

3) Sejam $x=\left(\begin{array}{ll}a & s \\ b & a\end{array}\right)$ e $y=\left(\begin{array}{cc}a^{\prime} & t \\ c & a^{\prime}\end{array}\right)$ dois elementos arbitrários de $B$, isto é, $s b=a^{2}-1$ e $t c=a^{\prime 2}-1$. Então

$$
x y x=\left(\begin{array}{cc}
a^{2} a^{\prime}+s c a+a b t+s b a^{\prime} & 2 a a^{\prime} s+s^{2} c+a^{2} t \\
2 b a a^{\prime}+a^{2} c+b^{2} t & a^{2} a^{\prime}+s c a+a b t+s b a^{\prime}
\end{array}\right)
$$

que é um elemento de $B$. Isto conclui a demonstração que $B$ é um subgrupo torcido de $G$.

Finalmente, note que

$$
B H=\left\{\left(\begin{array}{cc}
a r & b r^{-1} \\
s r & a r^{-1}
\end{array}\right), a=t s+1, b=t(2+t s), t, s \in \mathbb{K}\right\} .
$$

Então o elemento $g=\left(\begin{array}{cc}1 & -1 \\ 1 & 0\end{array}\right) \in G$ mas não pertence a $B H$ pois teríamos $a r^{-1}=0$, ou seja, $a=0$ e de $a r=-1$ obteríamos $-1=0$. Portanto, como $B H$ é um subconjunto próprio de $G$, segue que $B$ não é um loop de Bol algébrico.

\subsection{Loops de Bol algébricos de dimensão 2 sobre cor- pos que não são algebricamente fechados}

(I) O grupo algébrico da álgebra de Lie Lie $\mathfrak{B}(0,0, \gamma)$.

Caso 1: Se $\gamma=0$, então o resultado é análogo ao do caso quando $\mathbb{K}$ era algebricamente fechado.

Caso 2: Vamos supor que $\gamma \neq 0$. Neste caso, Lie $\mathfrak{B}(0,0, \gamma)$ tem base $\{v, w, d\}$ e produto dado por $[v, w]=-d,[v, d]=0,[w, d]=\gamma v$. 
Lema 3.2.1: 1) O conjunto

$$
G=\left\{\left(\begin{array}{ccc}
a & x & \gamma b \\
0 & 1 & 0 \\
b & y & a
\end{array}\right) \mid a, b, x, y \in \mathbb{K} \text { e } a^{2}-\beta b^{2}=1\right\}
$$

é um grupo algébrico.

2) A álgebra de Lie do grupo $G$ é

$$
\operatorname{Lie}(G)=\left\{\left(\begin{array}{ccc}
0 & z & \gamma y \\
0 & 0 & 0 \\
y & x & 0
\end{array}\right) \mid x, y, z \in \mathbb{K}\right\} .
$$

3) $\operatorname{Lie}(G) \simeq \operatorname{Lie} \mathfrak{B}(0,0, \gamma)$.

Demonstração: 1) Sejam $x=\left(\begin{array}{ccc}a_{1} & x_{1} & \gamma b_{1} \\ 0 & 1 & 0 \\ b_{1} & y_{1} & a_{1}\end{array}\right)$ e $y=\left(\begin{array}{ccc}a_{2} & x_{2} & \gamma b_{2} \\ 0 & 1 & 0 \\ b_{2} & y_{2} & a_{2}\end{array}\right)$ dois elementos arbitrários de $G$. Como

$$
x y=\left(\begin{array}{ccc}
a_{1} a_{2}+\gamma b_{1} b_{2} & a_{1} x_{2}+x 1+\gamma b_{1} b_{2} & \gamma\left(a_{1} b_{2}+b_{1} a_{2}\right) \\
0 & 1 & 0 \\
b_{1} a_{2}+a_{1} b_{2} & b_{1} a_{2}+y_{1} x_{2}+a 1 y_{2} & \gamma b_{1} b_{2}+a_{1} a_{2}
\end{array}\right) \in G
$$

concluímos que $G$ é fechado para a multiplicação.

Claramente $G$ é associativo. Tomando $a=1, b=x=0$ vemos que $E \in G$. Finalmente, seja $r=\left(\begin{array}{ccc}a & x & \gamma b \\ 0 & 1 & 0 \\ b & y & a\end{array}\right) \in G$. Como $r^{-1}=\left(\begin{array}{ccc}a & -x a+\gamma b y & -\gamma b \\ 0 & 1 & 0 \\ -b & -a y+x b & a\end{array}\right)$ também é um elemento de $G$, fica demonstrado que $G$ é de fato um grupo, com a multiplicação de matrizes.

2) Temos que $X=\left(x_{i j}\right) \in \operatorname{Lie}(G)$ se, e somente se, $E+\delta X \in G$. Então temos imediatamente que $x_{21}=x_{23}=0$. Como $1+\delta x_{22}=1$ segue que $x_{22}=0$. Como $X \in \operatorname{Lie}(G)$ devemos ter $x_{11}+x_{33}=0$ e como $1+\delta x_{11}=1+\delta x_{33}$ segue que 
$x_{11}-x_{33}=0$ donde concluímos que $x_{11}=x_{33}=0$. Finalmente temos que $x_{13}=\gamma x_{31}$. Isto prova que

$$
\operatorname{Lie}(G)=\left\{\left(\begin{array}{ccc}
0 & x_{12} & \gamma x_{31} \\
0 & 0 & 0 \\
x_{31} & x_{32} & 0
\end{array}\right) \mid x_{12}, x_{31}, x_{32} \in \mathbb{F}\right\} .
$$

3) Inicialmente, note que

$$
v=\left(\begin{array}{ccc}
0 & 0 & 0 \\
0 & 0 & 0 \\
0 & -1 & 0
\end{array}\right), w=\left(\begin{array}{ccc}
0 & 0 & \gamma \\
0 & 0 & 0 \\
1 & 0 & 0
\end{array}\right), d=\left(\begin{array}{ccc}
0 & -1 & 0 \\
0 & 0 & 0 \\
0 & 0 & 0
\end{array}\right)
$$

é uma base de $\operatorname{Lie}(G)$. A aplicação $T: \operatorname{Lie} \mathfrak{B}(0,0, \gamma) \rightarrow \operatorname{Lie}(G)$ definida por $T v=$ $v^{\prime}, T w=w^{\prime}, T d=\gamma d^{\prime}$ é um isomorfismo de álgebras de Lie.

Observação 3.2.2: 1) Pelo item 3) do Lema acima, $v, w$ e $d$ poderm ser representados por matrizes $3 \times 3$ sa seguinte forma:

$$
v=\left(\begin{array}{ccc}
0 & 0 & 0 \\
0 & 0 & 0 \\
0 & -1 & 0
\end{array}\right), w=\left(\begin{array}{ccc}
0 & 0 & \gamma \\
0 & 0 & 0 \\
1 & 0 & 0
\end{array}\right), d=\left(\begin{array}{ccc}
0 & -\gamma & 0 \\
0 & 0 & 0 \\
0 & 0 & 0
\end{array}\right)
$$

e, portanto

$$
\operatorname{Lie} \mathfrak{B}(0,0, \gamma) \simeq\left\{\left(\begin{array}{ccc}
0 & z & \gamma y \\
0 & 0 & 0 \\
y & x & 0
\end{array}\right) \mid x, y, z \in \mathbb{K}\right\}
$$

2)Note que $H=\left\{\exp \left(t^{\prime} d\right) \mid t^{\prime} \in \mathbb{K}\right\}=\left\{\left(\begin{array}{lll}1 & t & 0 \\ 0 & 1 & 0 \\ 0 & 0 & 1\end{array}\right) \mid t \in \mathbb{K}\right\}$ é um subgrupo algébrico de $G$. que

3) Como $w=\left(\begin{array}{ccc}0 & \sqrt{\gamma} & \sqrt{-\gamma} \\ 1 & 0 & 0 \\ 0 & 1 & 1\end{array}\right)\left(\begin{array}{ccc}0 & 0 & 0 \\ 0 & \sqrt{\gamma} & 0 \\ 0 & 0 & -\sqrt{\gamma}\end{array}\right)\left(\begin{array}{ccc}0 & 1 & 0 \\ \frac{1}{2 \sqrt{\gamma}} & 0 & \frac{1}{2} \\ -\frac{1}{2 \sqrt{\gamma}} & 0 & \frac{1}{2}\end{array}\right)$, segue 


$$
\begin{aligned}
\exp \left(s^{\prime} w\right) & =\left(\begin{array}{ccc}
0 & \sqrt{\gamma} & \sqrt{-\gamma} \\
1 & 0 & 0 \\
0 & 1 & 1
\end{array}\right)\left(\begin{array}{ccc}
1 & 0 & 0 \\
0 & s & 0 \\
0 & 0 & s^{-1}
\end{array}\right)\left(\begin{array}{ccc}
0 & 1 & 0 \\
\frac{1}{2 \sqrt{\gamma}} & 0 & \frac{1}{2} \\
-\frac{1}{2 \sqrt{\gamma}} & 0 & \frac{1}{2}
\end{array}\right) \\
& =\left(\begin{array}{ccc}
\frac{1}{2}\left(s+s^{-1}\right) & 0 & \frac{\sqrt{\gamma}}{2}\left(s-s^{-1}\right) \\
0 & 1 & 0 \\
\frac{1}{2 \sqrt{\gamma}}\left(s-s^{-1}\right) & 0 & \frac{1}{2}\left(s+s^{-1}\right)
\end{array}\right)=\left(\begin{array}{ccc}
a & 0 & \gamma b \\
0 & 1 & 0 \\
b & 0 & a
\end{array}\right),
\end{aligned}
$$

onde $a=\frac{1}{2}\left(s+s^{-1}\right)$ e $\frac{1}{2 \sqrt{\gamma}}\left(s-s^{-1}\right)$. Note também que exp $(t v)=\left(\begin{array}{ccc}1 & 0 & 0 \\ 0 & 1 & 0 \\ 0 & -t & 1\end{array}\right)$. Seja

$$
B=\left\{\left(\begin{array}{ccc}
1 & 0 & 0 \\
0 & 1 & 0 \\
0 & -t & 1
\end{array}\right)\left(\begin{array}{ccc}
a & 0 & \gamma b \\
0 & 1 & 0 \\
b & 0 & a
\end{array}\right)\left(\begin{array}{ccc}
1 & 0 & 0 \\
0 & 1 & 0 \\
0 & -t & 1
\end{array}\right) \mid a, b, t \in \mathbb{K} \text { e } a^{2}-\gamma b^{2}=1\right\}
$$

Portanto

$$
B=\left\{\left(\begin{array}{ccc}
a & -\gamma b t & \gamma b \\
0 & 1 & 0 \\
b & -t-a t & a
\end{array}\right) \mid a, b, t \in \mathbb{K} \text { e } a^{2}-\gamma b^{2}=1\right\}
$$

é uma subvariedade afim de $G$ cujo espaço tangente tem base $v$ e $w$.

Lema 3.2.3: A subvariedade $B$ construída no item 3) da observação acima é um subgrupo torcido de $G$, mas não é um subgrupo algébrico global.

Demonstração: (1) Pondo $a=1, b=0$ e $t=0$ vemos que o elemento identidade do grupo $G$ pertence a $B$.

(2) Seja $x=\left(\begin{array}{ccc}a & -\gamma b t & \gamma b \\ 0 & 1 & 0 \\ b & -t-a t & a\end{array}\right) \in B$. Como $x^{-1}=\left(\begin{array}{ccc}a & \gamma(-b) t & \gamma(-b) \\ 0 & 1 & 0 \\ -b & t+a t & a\end{array}\right)$, 
segue que $x^{-1} \in B$.

(3) Sejam $x=\left(\begin{array}{ccc}a & -\gamma b t & \gamma b \\ 0 & 1 & 0 \\ b & -t-a t & a\end{array}\right), y=\left(\begin{array}{ccc}c & -\gamma d s & \gamma d \\ 0 & 1 & 0 \\ d & -s-c s & c\end{array}\right)$ dois elementos arbitrários de $B$. Então $x y x=\left(a_{i j}\right) 1 \leq i, j \leq 3$, onde

$a_{11}=(a c+\gamma b d) a+(a d+b c) b \gamma$,

$a_{12}=-(a c+\gamma b d) \gamma b t-a d s \gamma-b t \gamma+(-s-c s) b \gamma+\gamma(a d+b c)(-t-a t)$,

$a_{13}=\gamma[(a c+b d \gamma) b+(a d+b c) a]$,

$a_{21}=0=a_{23}$ e $a_{22}=1$,

$a_{31}=(a c+b d \gamma) b+(a d+b c) a$,

$a_{32}=-(b c+a d) b t \gamma-b d s \gamma-t-a t+(-s-c s)+(a c+b d \gamma)(-t-a t)$,

$a_{33}=(a c+\gamma b d) a+(a d+b c) b \gamma$. Portanto, $B$ é um subgrupo torcido de $G$. Finalmente,

$$
B H=\left\{\left(\begin{array}{ccc}
a & -\gamma b t & \gamma b \\
0 & 1 & 0 \\
b & -t-a t & a
\end{array}\right)\left(\begin{array}{ccc}
1 & s & 0 \\
0 & 1 & 0 \\
0 & 0 & 1
\end{array}\right) \mid a, b, s, t \in \mathbb{K}, a^{2}-\gamma b^{2}=1\right\}
$$

isto é,

$$
B H=\left\{\left(\begin{array}{ccc}
a & a s-\gamma b t & \gamma b \\
0 & 1 & 0 \\
b & b s-t-a t & a
\end{array}\right) \mid a, b, s, t \in \mathbb{K}, a^{2}-\gamma b^{2}=1\right\} .
$$

Mas o elemento $X=\left(\begin{array}{ccc}-1 & 0 & 0 \\ 0 & 1 & 0 \\ 0 & 1 & -1\end{array}\right) \in G$ mas não pertence a $B H$. De fato, se $X \in B H$ então teríamos $a=-1, b=0$ e como neste caso $b s-t-a t=0$ o que implicaria $1=0$, o que é um absurdo. Portanto, $B H$ é um subconjunto próprio do grupo $G$ e isto mostra que $B$ não é um loop de Bol algébrico global.

(II) O grupo algébrico da álgebra de Lie $\operatorname{Lie} \mathcal{B}(1,0,0)$.

De acordo com o Lema (2.2.2) temos que $\operatorname{Lie} \mathcal{B}(1,0,0)$ tem base $v, w, d$ e multiplicação definida por $[v, w]=-d,[v, d]=-v,[w, d]=w$. Neste caso, a conclusão que chegamos é a mesma que no caso de $\mathbb{K}$ ser um corpo algebricamente fechado, isto é, 
que não existe um loop de Bol algébrico global.

(III) O grupo algébrico da álgebra de Lie $\operatorname{Lie} \mathcal{B}(0, \beta, 0)$.

CASO 1: Suponha que $\beta \in\left(\mathbb{K}^{*}\right)^{2}$. Neste caso, a álgebra de Lie Lie $\mathcal{B}(0, \beta, 0)$ é isomorfa a $\operatorname{Lie} \mathcal{B}(0,1,0)$, com base $\{v, w, d\}$ e relações definidas por: $[v, w]=-d,[v, d]=$ $1 w,[w, d]=0$. Fazendo $\beta=1$ no caso em que $\mathbb{K}$ é um corpo algebricamente fechado, obtemos a mesma conclusão anterior.

CASO 2: Suponha que $\beta \notin\left(\mathbb{K}^{*}\right)^{2}$. Considere a álgebra de Lie $\operatorname{Lie} \mathcal{B}(0, \beta, 0)$, com base $\{v, w, d\}$ e relações definidas por: $[v, w]=-d,[v, d]=-\beta w,[w, d]=0$.

Lema 3.2.4: 1) O conjunto

$$
G=\left\{\left(\begin{array}{ccc}
a & \beta b & x \\
b & a & y \\
0 & 0 & 1
\end{array}\right) \mid, x, y, b \in \mathbb{K}, a \in \mathbb{K}^{*}, \beta \notin\left(\mathbb{K}^{*}\right)^{2}, a^{2}-\beta b^{2}=1\right\}
$$

um grupo algébrico de dimensão 3 sobre $\mathbb{K}$.

2) A álgebra de Lie do grupo $G$ é

$$
\operatorname{Lie}(G)=\left\{\left(\begin{array}{ccc}
0 & \beta c & x \\
c & 0 & y \\
0 & 0 & 0
\end{array}\right) \mid c, x, y \in \mathbb{K}\right\} .
$$

3) $\operatorname{Lie}(G) \simeq \operatorname{Lie} \mathcal{B}(0, \beta, 0)$.

Demonstração: 1) Claramente $G$ é associativo. Tomando $a=1, b=x=y=0$, temos que $E \in G$. Sejam

$$
X=\left(\begin{array}{ccc}
a_{1} & \beta b_{1} & x_{1} \\
b_{1} & a_{1} & y_{1} \\
0 & 0 & 1
\end{array}\right), Y=\left(\begin{array}{ccc}
a_{2} & \beta b_{2} & x_{2} \\
b_{2} & a_{2} & y_{2} \\
0 & 0 & 1
\end{array}\right)
$$

dois elementos quaisquer de $G$. Como

$$
X Y=\left(\begin{array}{ccc}
a_{1} a_{2}+\beta b_{1} b_{2} & \beta\left(b_{1} a_{2}+a_{1} b_{2}\right) & a_{1} x_{2}+\beta b_{1} y_{2}+x_{1} \\
b_{1} a_{2}+a_{1} b_{2} & a_{1} a_{2}+\beta b_{1} b_{2} & b_{1} x_{2}+a_{1} y_{2}+y_{1} \\
0 & 0 & 1
\end{array}\right),
$$


segue que $G$ é fechado para a multiplicação. Finalmente, seja $X=\left(\begin{array}{ccc}a & \beta b & x \\ b & a & y \\ 0 & 0 & 1\end{array}\right)$ um elemento qualquer de $G$. Como $X^{-1}=\left(\begin{array}{ccc}a & -\beta b & -a x+\beta b y \\ -b & a & -a y+b x \\ 0 & 0 & 1\end{array}\right)$ é um elemento de $G$, temos que $G$ é um grupo.

2) Vamos determinar $\operatorname{Lie}(G)$. Lembremos que $X \in \operatorname{Lie}(G)$ se, e somente se, $E+\delta X \in$ $G$. O grupo $G$ é completamente determinado pelas seguintes equações:

$$
x_{31}=x_{32}=0, x_{33}=1, x_{11}=x_{22}, x_{11}^{2}-\beta x_{22}^{2}=1, x_{13}, x_{23} \in \mathbb{F} .
$$

Seja $X=\left(a_{i j}\right)_{1 \leq i, j \leq 3} \in \operatorname{Lie}(G)$. Então temos que

$$
E+\delta X=\left(\begin{array}{ccc}
1+\delta a_{11} & \delta a_{12} & \delta a_{13} \\
\delta a_{21} & 1+\delta a_{22} & \delta a_{23} \\
\delta a_{31} & \delta a_{32} & 1+\delta a_{33}
\end{array}\right) \in G
$$

se, e somente se,

$$
\delta a_{31}=\delta a_{32}=0,1+\delta a_{33}=1,1+\delta a_{11}=1+\delta a_{22} \text { e }\left(1+\delta a_{11}\right)\left(1+\delta a_{22}\right)=1 .
$$

Mas estas equações são satisfeitas se, e somente se, $a_{11}=a_{22}=a_{31}=a_{32}=a_{33}=0$. Portanto,

$$
\operatorname{Lie}(G)=\left\{\left(\begin{array}{ccc}
0 & \beta c & x \\
c & 0 & y \\
0 & 0 & 0
\end{array}\right) \mid c, x, y \in \mathbb{F}\right\}
$$

3) Vamos mostrar agora que $\operatorname{Lie}(G)$ e $\operatorname{Lie} \mathcal{B}(0, \beta, 0)$ são isomorfas. Para ver isto, seja

$$
v^{\prime}=\left(\begin{array}{ccc}
0 & \beta & 0 \\
1 & 0 & 0 \\
0 & 0 & 0
\end{array}\right), w^{\prime}=\left(\begin{array}{ccc}
0 & 0 & 1 \\
0 & 0 & 0 \\
0 & 0 & 0
\end{array}\right), d^{\prime}=\left(\begin{array}{ccc}
0 & 0 & 0 \\
0 & 0 & -1 \\
0 & 0 & 0
\end{array}\right)
$$

uma base de $\operatorname{Lie}(G)$. Então a aplicação $T: \operatorname{Lie}(G) \rightarrow \operatorname{Lie} \mathcal{B}(0, \beta, 0)$ definida por $T v=v^{\prime}, T w=w^{\prime}, T d=d^{\prime}$ é um isomorfismo de álgebras de Lie. 
Observação 3.2.5: 1) Pelo item 3) do Lema anterior, $v, w, d$ podem ser representados por matrizes $3 \times 3$, com entradas no corpo $\mathbb{K}$ da seguinte forma:

$$
v=\left(\begin{array}{ccc}
0 & \beta & 0 \\
1 & 0 & 0 \\
0 & 0 & 0
\end{array}\right), w=\left(\begin{array}{lll}
0 & 0 & 1 \\
0 & 0 & 0 \\
0 & 0 & 0
\end{array}\right), d=\left(\begin{array}{ccc}
0 & 0 & 0 \\
0 & 0 & -1 \\
0 & 0 & 0
\end{array}\right) .
$$

2)Temos que

$$
H=\left\{\exp \left(t^{\prime} d\right) \mid t^{\prime} \in \mathbb{K}\right\}=\left\{\left(\begin{array}{ccc}
1 & 0 & \beta t \\
0 & 1 & -t \\
0 & 0 & 1
\end{array}\right) \mid t \in \mathbb{K}\right\}
$$

é um subgrupo algébrico de $G$.

3) Inicialmente notemos que $\exp (s w)=\left(\begin{array}{lll}1 & 0 & s \\ 0 & 1 & 0 \\ 0 & 0 & 1\end{array}\right)$. Como

$$
v=\left(\begin{array}{ccc}
0 & \beta & 0 \\
1 & 0 & 0 \\
0 & 0 & 0
\end{array}\right)=\left(\begin{array}{ccc}
0 & \sqrt{\beta} & -\sqrt{\beta} \\
0 & 1 & 1 \\
1 & 0 & 0
\end{array}\right)\left(\begin{array}{ccc}
0 & 0 & 0 \\
0 & \sqrt{\beta} & 0 \\
0 & 0 & -\sqrt{\beta}
\end{array}\right)\left(\begin{array}{ccc}
0 & 0 & 1 \\
\frac{1}{2 \sqrt{\beta}} & \frac{1}{2} & 0 \\
-\frac{1}{2 \sqrt{\beta}} & \frac{1}{2} & 0
\end{array}\right)
$$

temos que

$$
\begin{aligned}
\exp \left(t^{\prime} v\right) & =\left(\begin{array}{ccc}
0 & \sqrt{\beta} & -\sqrt{\beta} \\
0 & 1 & 1 \\
1 & 0 & 0
\end{array}\right)\left(\begin{array}{ccc}
1 & 0 & 0 \\
0 & t & 0 \\
0 & 0 & t^{-1}
\end{array}\right)\left(\begin{array}{ccc}
0 & 0 & 1 \\
\frac{1}{2 \sqrt{\beta}} & \frac{1}{2} & 0 \\
-\frac{1}{2 \sqrt{\beta}} & \frac{1}{2} & 0
\end{array}\right), t \in \mathbb{K}^{*} \\
& =\left(\begin{array}{ccc}
\frac{1}{2}\left(t+t^{-1}\right) & \frac{\sqrt{\beta}}{2}\left(t-t^{-1}\right) & 0 \\
\frac{1}{2 \sqrt{\beta}}\left(t-t^{-1}\right) & \frac{1}{2}\left(t+t^{-1}\right) & 0 \\
0 & 0 & 1
\end{array}\right)
\end{aligned}
$$

$\mathrm{e} t=\exp \left(t^{\prime} \sqrt{\beta}\right)$.

$$
\text { Pondo } a=\frac{1}{2}\left(t+t^{-1}\right) \text { e } b=\frac{1}{2 \sqrt{\beta}}\left(t-t^{-1}\right) \text {, temos que } \exp \left(t^{\prime} v\right)=\left(\begin{array}{ccc}
a & \beta b & 0 \\
b & a & 0 \\
0 & 0 & 1
\end{array}\right) \text {, }
$$

onde $a^{2}-\beta b^{2}=1$. Então 


$$
\begin{aligned}
B & =\{\exp (s w) \exp (t v) \exp (s w) \mid r, s \in \mathbb{K}\} \\
& =\left\{\left(\begin{array}{lll}
1 & 0 & s \\
0 & 1 & 0 \\
0 & 0 & 1
\end{array}\right)\left(\begin{array}{ccc}
a & \beta b & 0 \\
b & a & 0 \\
0 & 0 & 1
\end{array}\right)\left(\begin{array}{ccc}
1 & 0 & s \\
0 & 1 & 0 \\
0 & 0 & 1
\end{array}\right) \mid s, a, b \in \mathbb{K} \text { e } a^{2}-\beta b^{2}=1\right\} \\
& =\left\{\left(\begin{array}{ccc}
a & \beta b & a s+s \\
b & a & b s \\
0 & 0 & 1
\end{array}\right) \mid s, a, b \in \mathbb{F} \mathrm{e} a^{2}-\beta b^{2}=1\right\}
\end{aligned}
$$

é uma subvariedade afim de $G$ de dimensão 2 tendo $v$ e $w$ como vetores tangentes.

Lema 3.2.6: A subvariedade $B$, construída acima é um subgrupo torcido do grupo $G$, que não é um loop de Bol algébrico global.

Demonstração: (1) Pondo $a=1, b=0, s=0$, vemos que o elemento neutro do grupo $G$ pertence a $B$.

(2) Seja $x=\left(\begin{array}{ccc}a & \beta b & a s+s \\ b & a & b s \\ 0 & 0 & 1\end{array}\right)$ um elemento qualquer de $B$. Então o elemento $x^{-1}=\left(\begin{array}{ccc}a & \beta b & -s(a+\beta b+1) \\ b & a & -s(-b-a-1) \\ 0 & 0 & 1\end{array}\right)$ é um elemento de $B$.

(3) Sejam $x=\left(\begin{array}{ccc}a & \beta b & a s+s \\ b & a & b s \\ 0 & 0 & 1\end{array}\right)$ e $y=\left(\begin{array}{ccc}c & \beta d & c t+t \\ d & c & d t \\ 0 & 0 & 1\end{array}\right)$ dois elementos arbitrários de $B$. Então

$$
x y x=\left(\begin{array}{ccc}
(a c+\beta b d) a+(a d+b c) \beta b & \beta((a c+\beta b d) b+(a d+b c) a) & a_{13} \\
(b c+a d) a+(a c+\beta b d) b & (b c+a d) \beta b+(a c+\beta b d) a & a_{23} \\
0 & 0 & 1
\end{array}\right),
$$

onde $a_{13}=(a c+\beta b d)(a s+s)+\beta b s(a d+b c)+a c t+a t+\beta b d t$ e $a_{23}=(b c+a d)(a s+$ 
$s)+(\beta b d+a c) b s+b c t+b t+a d t$. Disto concluímos que $B$ é um subgrupo torcido de $G$.

Finalmente, temos que

$$
\begin{aligned}
B H & =\left\{\left(\begin{array}{ccc}
a & b \beta & a s+s \\
b & a & b s \\
0 & 0 & 1
\end{array}\right)\left(\begin{array}{ccc}
1 & 0 & 0 \\
0 & 1 & -t \\
0 & 0 & 1
\end{array}\right) \mid s, a, b \in \mathbb{K} \mathrm{e} a^{2}-\beta b^{2}=1\right\} \\
& =\left\{\left(\begin{array}{ccc}
a & \beta b & -\beta b t+a s+s \\
b & a & -a t+b s \\
0 & 0 & 1
\end{array}\right) \mid s, a, b \in \mathbb{K} \mathrm{e} a^{2}-\beta b^{2}=1\right\} .
\end{aligned}
$$

Agora o elemento $X=\left(\begin{array}{ccc}-1 & 0 & 0 \\ 0 & -1 & 0 \\ 0 & 0 & 1\end{array}\right)$ pertence a $G$ mas não pertence a $B H$.

De fato, se $X \in B H$ teríamos

$$
\left(\begin{array}{ccc}
-1 & 0 & 0 \\
0 & -1 & 0 \\
0 & 0 & 1
\end{array}\right)=\left(\begin{array}{ccc}
a & \beta b & -\beta b t+a s+s \\
b & a & -a t+b s \\
0 & 0 & 1
\end{array}\right)
$$

Daí teríamos $a=-1, b=0$ e $a s+s=-a+s=0$, ou seja, $1=0$, o que é um absurdo. Portanto $B H$ é um subconjunto próprio de $G$ e isto mostra que $B$ não é um loop de Bol algébrico global.

(IV) O grupo algébrico da álgebra de Lie $\operatorname{Lie} \mathcal{B}(0, \beta, \gamma)$.

Caso 1: $\beta \neq 0$ e $\gamma \neq 0$. Neste caso $\operatorname{Lie}(0, \beta, \gamma)$ tem base $\{v, w, d\}$ e produto dado por $[v, w]=-d,[v, d]=-\beta w,[w, d]=\gamma v$. Seja $\mathbf{Q}(a, b)$ a álgebras dos quatérnios sobre o corpo $\mathbb{K}$, onde $a, b \in \mathbb{K}$, tendo base $1, v, w, u$ e a multiplicação desses elementos é definida da seguinte maneira:

$$
v^{2}=a, w^{2}=b, v w=-w v=u \text {. }
$$

Daqui concluímos que $u u=-a b .1, u w=b v, w u=-b v, v u=a w, u v=-a w$.

Vamos representar $\mathbf{Q}(a, b)$ como matrizes $4 \times 4$ com entradas no corpo $\mathbb{K}$. Para isto seja $t=t_{1}+t_{2} v+t_{3} w+t_{4} u$ um elemento arbitrário de $\mathbf{Q}(a, b)$. Então 


$$
\begin{aligned}
& R_{t}(1)=t .1=t_{1}+t_{2} v+t_{3} w+t_{4} u \\
& R_{t}(v)=v t=t_{1} v+t_{2} v v+t_{3} v w+t_{4} v u=t_{1} v+\left(a t_{2}\right) .1+t_{3} u+a t_{4} w \\
& =\left(a t_{2}\right) .1+t_{1} v+a t_{4} w+t_{3} . \\
& R_{t}(w)=w t=t_{1} w+t_{2} w v+t_{3} w w+t_{4} w u=t_{1} w-t_{2} u+b t_{3}-b t_{4} v \\
& =b t_{3}-b t_{4} v+t_{1} w-t_{2} u . \\
& R_{t}(u)=u t=t_{1} u+t_{2} u v+t_{3} u w+t_{4} u u=t_{1} u-a t_{2} w+b t_{3} v-a b t_{4} 1 \\
& =-a b t_{4}+t_{3} b v-a t_{2} w+t_{1} u .
\end{aligned}
$$

Logo obtemos a seguinte representação de $\mathbf{Q}(a, b)$.

$$
t=t_{1}+t_{2} v+t_{3} w+t_{4} u \rightarrow\left(\begin{array}{cccc}
t_{1} & a t_{2} & b t_{3} & -a b t_{4} \\
t_{2} & t_{1} & -b t_{4} & b t_{3} \\
t_{3} & a t_{4} & t_{1} & -a t_{2} \\
t_{4} & t_{3} & -t_{2} & t_{1}
\end{array}\right)
$$

Vamos determinar a álgebra de Lie do grupo algébrico formado pelas matrizes da forma acima com determinante igual a 1 .

$$
\begin{gathered}
\text { Então } X=\left(\begin{array}{cccc}
x_{11} & x_{12} & x_{13} & x_{14} \\
x_{21} & x_{22} & x_{23} & x_{24} \\
x_{31} & x_{32} & x_{33} & x_{34} \\
x_{41} & x_{42} & x_{43} & x_{44}
\end{array}\right) \in \operatorname{Lie}(G) \text { se, e somente se, } \\
\left(\begin{array}{cccc}
1+\delta x_{11} & x_{12} & x_{13} & x_{14} \\
x_{21} & 1+\delta x_{22} & x_{23} & x_{24} \\
x_{31} & x_{32} & 1+\delta x_{33} & x_{34} \\
x_{41} & x_{42} & x_{43} & 1+\delta x_{44}
\end{array}\right) \in G .
\end{gathered}
$$

Daí concluímos que $x_{11}=x_{22}=x_{33}=x_{44}$ e como os elementos de Lie $(G)$ devem ter traço 0, temos que a sua diagonal é constituída de zeros. Finalmente conclui-se que 
$X=\left(\begin{array}{cccc}0 & a x_{21} & b x_{31} & -a b \\ x_{21} & 0 & -b x_{41} & b x_{31} \\ x_{31} & a x_{41} & 0 & -a x_{21} \\ x_{41} & x_{31} & -x_{21} & 0\end{array}\right)$. Logo
$\operatorname{Lie}(G)=\left\{\left(\begin{array}{cccc}0 & a x_{21} & b x_{31} & -a b x_{41} \\ x_{21} & 0 & -b x_{41} & b x_{31} \\ x_{31} & a x_{41} & 0 & -a x_{21} \\ x_{41} & x_{31} & -x_{21} & 0\end{array}\right) \mid a, b, x_{21}, x_{21}, x_{21} \in \mathbb{K}\right.$ e $a, b$ não são quadrados $\}$.

Seja

$$
v^{\prime}=\left(\begin{array}{cccc}
0 & a & 0 & 0 \\
1 & 0 & 0 & 0 \\
0 & 0 & 0 & -a \\
0 & 0 & -1 & 0
\end{array}\right), w^{\prime}=\left(\begin{array}{cccc}
0 & 0 & b & 0 \\
0 & 0 & 0 & b \\
1 & 0 & 0 & 0 \\
0 & 1 & 0 & 0
\end{array}\right), d^{\prime}=\left(\begin{array}{cccc}
0 & 0 & 0 & -a b \\
0 & 0 & -b & 0 \\
0 & a & 0 & 0 \\
1 & 0 & 0 & 0
\end{array}\right)
$$

uma base de $\operatorname{Lie}(G)$. Vemos que $\left[v^{\prime}, w^{\prime}\right]=-2 d^{\prime},\left[v^{\prime}, d^{\prime}\right]=-2 a w,\left[w^{\prime}, d^{\prime}\right]=2 b v$. Pondo $a=\beta$ e $b=4 \gamma$ temos que a aplicação $T: \operatorname{Lie} \mathcal{B}(0, \beta, \gamma) \rightarrow \operatorname{Lie}(G)$ dada por

$$
T v=\frac{v^{\prime}}{2}, T w=w^{\prime}, T d=d^{\prime}
$$

é um isomorfismo de álgebras de Lie e então representar $v, w$ e $d$ como matrizes $4 \times 4$ sobre o corpo $\mathbb{K}$ como:

$$
v=\left(\begin{array}{cccc}
0 & \beta & 0 & 0 \\
1 & 0 & 0 & 0 \\
0 & 0 & 0 & -\beta \\
0 & 0 & -1 & 0
\end{array}\right), w=\left(\begin{array}{cccc}
0 & 0 & 4 \gamma & 0 \\
0 & 0 & 0 & 4 \gamma \\
1 & 0 & 0 & 0 \\
0 & 1 & 0 & 0
\end{array}\right), d=\left(\begin{array}{cccc}
0 & 0 & 0 & -4 \beta \gamma \\
0 & 0 & -4 \gamma & 0 \\
0 & \beta & 0 & 0 \\
1 & 0 & 0 & 0
\end{array}\right)
$$


Pondo $t_{1}=\frac{1}{2}\left(e^{\sqrt{-\alpha \beta} t}+e^{-\sqrt{-\alpha \beta} t}\right)$ e $t_{2}=\frac{\alpha \beta}{2 \sqrt[3]{-\alpha \beta}}\left(e^{\sqrt{-\alpha \beta} t}-e^{-\sqrt{-\alpha \beta} t}\right)$ temos que

$$
H=\{\exp (r d) ; r \in \mathbb{K}\}=\left\{\left(\begin{array}{cccc}
r_{1} & 0 & 0 & \alpha \beta r_{2} \\
0 & r_{1} & \gamma r_{2} & 0 \\
0 & \alpha r_{2} & r_{1} & 0 \\
-r_{1} & 0 & 0 & r_{1}
\end{array}\right)\right\}
$$

Pondo $r_{1}=\frac{1}{2}\left(e^{\sqrt{\alpha} r}+e^{-\sqrt{\alpha} r}\right), r_{2}=\frac{-1}{2 \sqrt{\beta}} 2\left(e^{\sqrt{\alpha} r}-e^{-\sqrt{\alpha} r}\right), s_{1}=\frac{1}{2}\left(e^{2 \sqrt{\gamma} t}+e^{-2 \sqrt{\gamma} t}\right) \mathrm{e}$ $s_{2}=\frac{1}{\sqrt{\gamma}}\left(e^{2 \sqrt{\gamma} t}-e^{-2 \sqrt{\gamma} t}\right)$ temos que

$$
\exp (t v)=\left(\begin{array}{cccc}
t_{1} & \beta t_{2} & 0 & 0 \\
t_{2} & t_{1} & 0 & 0 \\
0 & 0 & t_{1} & -\beta t_{2} \\
0 & 0 & -t_{2} & t_{1}
\end{array}\right) \text { e } \exp (s w)=\left(\begin{array}{cccc}
s_{1} & 0 & \gamma s_{2} & 0 \\
0 & s_{1} & 0 & \gamma s_{2} \\
\frac{s_{2}}{4} & 0 & s_{1} & 0 \\
0 & \frac{s_{2}}{4} & 0 & s_{1}
\end{array}\right) .
$$

Portanto,

$$
H=\{\exp (r v) \exp (s w) \exp (r v) ; r, s \in \mathbb{K}\}=\left\{\left(\begin{array}{cccc}
t_{1} & \beta t_{2} & \gamma t_{2} & 0 \\
t_{2} & t_{1} & 0 & \gamma t_{3} \\
\frac{t_{3}}{4} & 0 & t_{1} & -\beta t_{2} \\
0 & \frac{t_{3}}{4} & -t_{2} & t_{1}
\end{array}\right)\right\} .
$$

Lema 3.2.7: A subvariedade $B$ construída acima é um subgrupo torcido de $G$ que não é um loop de Bol algébrico global.

Demonstração: Inicialmente, vemos que o elemento identidade pertence a $B$. Se $x=\left(\begin{array}{cccc}t_{1} & \beta t_{2} & \gamma t_{2} & 0 \\ t_{2} & t_{1} & 0 & \gamma t_{3} \\ \frac{t_{3}}{4} & 0 & t_{1} & -\beta t_{2} \\ 0 & \frac{t_{3}}{4} & -t_{2} & t_{1}\end{array}\right)$ é um elemento arbitrário de $B$, então temos que 
$x^{-1}=\left(\begin{array}{cccc}4 t_{1} & -4 \beta t_{2} & -4 \gamma t_{2} & 0 \\ -4 t_{2} & 4 t_{1} & 0 & -4 \gamma t_{3} \\ -t_{3} & 0 & 4 t_{1} & 4 \beta t_{2} \\ 0 & -t_{3} & 4 t_{2} & 4 t_{1}\end{array}\right)$ também é um elemento de B. Finalmente, sejam

$$
x=\left(\begin{array}{cccc}
t_{1} & \beta t_{2} & \gamma t_{2} & 0 \\
t_{2} & t_{1} & 0 & \gamma t_{3} \\
\frac{t_{3}}{4} & 0 & t_{1} & -\beta t_{2} \\
0 & \frac{t_{3}}{4} & -t_{2} & t_{1}
\end{array}\right) \text { e } y=\left(\begin{array}{cccc}
s_{1} & \beta s_{2} & \gamma s_{2} & 0 \\
s_{2} & s_{1} & 0 & \gamma s_{3} \\
\frac{s_{3}}{4} & 0 & s_{1} & -\beta s_{2} \\
0 & \frac{s_{3}}{4} & -s_{2} & s_{1}
\end{array}\right)
$$

dois elementos arbitrários de $B$. Então calculando $x y x$, vemos que ainda é um elemento de $B$.

$$
\text { Como o elemento }\left(\begin{array}{cccc}
-1 & 0 & 0 & 0 \\
0 & -1 & 0 & 0 \\
0 & 0 & -1 & 0 \\
0 & 0 & 0 & -1
\end{array}\right) \text { pertence a } B \cap H \text { e é diferente do ele- }
$$

mento identidade de $G$ temos que $B$ não é um loop de Bol algébrico global.

Finalmente, estamos em condições de provar o resultado principal deste capítulo.

Teorema 3.2.8: Todo loop de Bol analítico (algébrico) global sobre um corpo de característica 0 é um grupo.

Demonstração: Pela Proposição 1.4.30, se $\mathcal{B}$ é uma álgebra de Bol, então existe um par de álgebras de Lie $(\mathcal{G}, \mathcal{H})$ tais que $\mathcal{G}=\mathcal{M} \oplus \mathcal{H}$. A aplicação exponencial nos dá

$$
G=\exp (\mathcal{G})=\exp (\mathcal{M}) \exp (\mathcal{H})=B H
$$

, onde $H$ é um subgrupo fechado de $G$ e, desde que $\mathcal{B}$ é uma álgebra de Bol, $B$ é um subgrupo torcido. Pelo Teorema 1.5.4 temos que $(B, *)$ é um loop de Bol analítico local. Se ele fosse global, teríamos que $B$ seria um conjunto de representantes das classes laterais de $H$ em $G$ o que não é verdade pelo que vimos anteriormente. 


\section{Capítulo 4}

\section{Loops de Bol Algébricos Globais e Álgebras de Bol Críticas}

No início desta tese tinhamos como principal objetivo decidir se a conjectura abaixo, devido a G. Nagy, era verdadeira ou falsa.

Conjectura: Seja $B$ um loop de Bol algébrico sobre um corpo algebricamente fechado de característica 0 . Se $R M l t(B)$ é nilpotente, então $B$ é nilpotente.

Na primeira seção deste capítulo, exibiremos um contra-exemplo para a conjectura.

\subsection{Loops de Bol Algébricos}

Seja $V$ um espaço vetorial de dimensão $n$ sobre $\mathbb{K}$ e $X \subset \operatorname{End}(V)$ consistindo de elementos nilpotentes. Em particular, $x^{n}=0$ para todo $x \in X$ e a aplicação

$$
x \mapsto(1-x)^{-1}=1+x+\cdots+x^{n-1}
$$

é um $\mathbb{K}$-morfismo de $X$ em $\operatorname{End}(V)$.

O Lema abaixo será necessário para exibirmos um loop de Bol algébrico global de dimensão 3.

Lema 4.1.1: Sejam $\mathbb{K}$ um corpo algebricamente fechado tal que $\operatorname{char}(\mathbb{K}) \neq 2$ e $X$ um subespaço vetorial das matrizes com entradas em $\mathbb{K}$. Então $(2-x)^{-1}$ existe para todo $x \in X$ se, e somente se, $X$ contém apenas elementos nilpotentes. 
Demonstração: Suponha que exista $x \in X$ não nilpotente. Como $K$ é algebricamente fechado, existe $\lambda \neq 0$ em $\mathbb{K}$ e $v \in V$ tal que $x v=\lambda v$. Neste caso, temos $\left(2-\frac{2}{\lambda} x\right) v=0$. A recíproca é trivial.

Lema 4.1.2: Seja $V$ um espaço vetorial sobre $\mathbb{K}$ de dimensão $n$ e $X$ um subespaço vetorial de $\operatorname{End}(V)$ consistindo de elementos nilpotentes. Seja $G=X \oplus V \oplus V$ tal que

$$
\left(x_{1}, v_{1}, w_{1}\right)\left(x_{2}, v_{2}, w_{2}\right)=\left(x_{1}+x_{2}, v_{1}+v_{2}, w_{1}+w_{2}+\frac{1}{2}\left(x_{1} v_{2}-x_{2} v_{1}\right)\right)
$$

para quaisquer $\left(x_{i}, v_{i}, w_{i}\right) \in G, i=1,2$, é um grupo algébrico 2-nilpotente.

Demonstração: Um cálculo direto mostra que a operação definida acima é associativa. O elemento neutro é $(0,0,0)$ e, se $(x, v, w)$ é um elemento arbitrário de $G$, então $(x, v, w)^{-1}=(-x,-v,-w)$. Note que

$$
\left[\left(x_{1}, v_{1}, w_{1}\right),\left(x_{2}, v_{2}, w_{2}\right)\right]=\left(0,0, x_{1} v_{2}-x_{2} v_{1}\right)
$$

e, portanto, temos que $G$ é um grupo 2-nilpotente.

Lema 4.1.3: Seja $G$ o grupo algébrico do Lema anterior.

1) O subconjunto $K=\{(x, v, 0) \mid x \in X, v \in V\}$ é um subgrupo torcido de $G$.

2) O subconjunto $H=\{(0, w, w) \mid w \in V\}$ é um subgrupo algébrico de $G$ isomorfo a $(V,+)$.

3) $G=H K$ e $H \cap K=\{0\}$.

4) A operação

$$
\left(x_{1}, v_{1}\right)\left(x_{2}, v_{2}\right)=\left(x_{1}+x_{2}, v_{1}+v_{2}-\left(2-x_{1}-x_{2}\right)^{-1}\left(x_{1} v_{2}-x_{2} v_{1}\right)\right)
$$

define uma estrutura de loop de Bol algébrico global em $K$.

Demonstração: 1) Sejam $\left(x_{1}, v_{1}, 0\right),\left(x_{2}, v_{2}, 0\right)$ dois elementos arbitrários de $K$. Então como

$$
\left(x_{1}, v_{1}, 0\right)\left(x_{2}, v_{2}, 0\right)\left(x_{1}, v_{1}, 0\right)=\left(2 x_{1}+x_{2}, 2 v_{1}+v_{2}, 0\right) \in K
$$


temos que $K$ é, por definição, um subgrupo torcido de $G$.

2) Vê-se facilmente que $H$ é um subgrupo algébrico de $G$.

3) Segue das definições de $H$ e $K$ que $H \cap K=\{0\}$. Temos $\left(0,\left(1-\frac{x}{2}\right)^{-1} w,\left(1-\frac{x}{2}\right)^{-1} w\right) \in$ $H$ e $\left(x, v-\left(1-\frac{x}{2}\right)^{-1} w, 0\right) \in K$ e, além disso,

$$
(x, v, w)=\left(0,\left(1-\frac{x}{2}\right)^{-1} w,\left(1-\frac{x}{2}\right)^{-1} w\right)\left(x, v-\left(1-\frac{x}{2}\right)^{-1} w, 0\right) .
$$

4) Vamos determinar a multiplicação entre dois elementos de $K$. Note que para $\left(x_{1}, v_{1}, 0\right),\left(x_{2}, v_{2}, 0\right) \in G$ temos por um lado,

$$
\left(x_{1}, v_{1}, 0\right)\left(x_{2}, v_{2}, 0\right)=(0, w, w)(x, v, 0)=\left(x, w+v, w-\frac{1}{2} x w\right)
$$

e, por outro lado temos

$$
\left(x_{1}, v_{1}, 0\right)\left(x_{2}, v_{2}, 0\right)=(0, w, w)(x, v, 0)=\left(x_{1}+x_{2}, v_{1}+v_{2}, \frac{1}{2}\left(x_{1} v_{2}-x_{2} v_{1}\right)\right) .
$$

Daí temos, $x=x_{1}+x_{2}, w+v=v_{1}+v_{2}$ e $\left(1-\frac{x}{2}\right) w=\frac{1}{2}\left(x_{1} v_{2}-x_{2} v_{1}\right)$ o que nos dá $w=\left(2-x_{1}-x_{2}\right)^{-1}\left(x_{1} v_{2}-x_{2} v_{1}\right)$. Observe que o Lema 4.1 .1 garante que as matrizes $1-\frac{x}{2}$ e $2-x_{1}-x_{2}$ são inversíveis.

Vamos utilizar os lemas anteriores para dar um contra-exemplo a conjectura enunciada anteriormente.

Exemplo 4.1.4: Ponha $V=\mathbb{K}^{3} \mathrm{e}$

$$
X=\left\{\left(\begin{array}{ccc}
0 & s & 0 \\
t & 0 & s \\
0 & -t & 0
\end{array}\right) s, t \in \mathbb{K}\right\} .
$$

Note que $X$ é constituído de elementos nilpotentes. Então temos um loop de Bol algébrico global $L=X \oplus \mathbb{K}^{3}$ com a multiplicação definida no item 4 do Lema anterior. Vamos mostrar que $L$ não é nilpotente mostrando que $\mathcal{Z}(L)=\{0\}$. Para qualquer $x_{1} \in X$ não-nulo existe um $v_{2} \in V$ tal que $x_{1} v_{2} \neq 0$ e para qualquer $v_{1} \in V$ não-nulo existe um $x_{2} \in X$ tal que $x_{2} v_{1} \neq 0$. Portanto, por (3) $\left(x_{1}, v_{1}\right)$ não comuta com $\left(0, v_{2}\right)$ e $\left(0, v_{1}\right)$ não comuta com $\left(x_{2}, 0\right)$. Isto significa que o centro do loop de Bol algébrico correspondente é trivial. Portanto, $B$ não é um loop de Bol nilpotente. 
Nosso próximo passo é construir, para todo $n \in \mathbb{N}, n \geq 3$ loops de Bol algébricos globais de dimensão $n$.

Sejam $V$ um espaço vetorial sobre um corpo algebricamente fechado $\mathbb{K}, \operatorname{char}(\mathbb{K}) \neq$ 2, $X \subset \operatorname{End}(V)$ e $\bar{V}$ um espaço vetorial isomorfo a $V$ e seja $\varphi$ um isomorfismo de $V$ em $\bar{V}$. Considere o espaço vetorial $L=X \oplus V \oplus \bar{V}$. Em $L$ definimos a seguinte operação binária:

$$
\left[x_{1}+v_{1}+\overline{w_{1}}, x_{2}+v_{2}+\overline{w_{2}}\right]=\varphi\left(x_{2} v_{1}-x_{1} v_{2}\right)
$$

Algumas vezes escreveremos $\bar{v}$ para denotar o elemento $\phi(v)$.

Lema 4.1.5: (1) Com a multiplicação definida acima $L$ é uma álgebra de Lie nilpotente de índice 2.

(2) $Z(L)=\bar{V}$.

(3) A aplicação $\sigma: L \rightarrow L$ definida por $\sigma u=-u$ se $u \in X \oplus V$ e $\sigma u=u$ se $u \in \bar{V}$ é um automorfismo involutivo.

(4) $L=(X \oplus V) \oplus\{v+\bar{v} \mid v \in V\}$.

(5) $H=\{v+\bar{v} \mid v \in V\}$ é uma subálgebra de $L$.

(6) $\mathcal{B}=X \oplus V$ com a operação $\left(x_{1}+v_{1}\right)\left(x_{2}+v_{2}\right)=-\left(x_{2} v_{1}-x_{1} v_{2}\right)$ é uma álgebra de Bol.

(7) A álgebra de Bol $B=V \oplus X$ é nilpotente se, e somente se, a álgebra associativa gerada por $X$ é nilpotente.

Demonstração: Os items 1), 3), 4), 5) e 6) são imediatos.

2) Seja $x+v+\bar{w} \in \mathcal{Z}(L)$ e $x_{1} \in L$. Então $0=\left[x+v+\bar{w}, x_{1}\right]=\overline{x_{1} v}$. Como $\varphi$ é um isomorfismo temos que $x_{1} v=0$ para todo $x_{1} \in L$. Logo $v=0$. Analogamente podemos provar que $x=0$ e, portanto $\mathcal{Z}(L) \subset \bar{V}$. A inclusão contrária é imediata.

Lema 4.1.6: (1) Em $G(L)=X \oplus V \oplus \bar{V}$ definimos uma operação binária da seguinte forma:

$$
\left(x_{1}+v_{1}+\overline{w_{1}}\right)\left(x_{2}+v_{2}+\overline{w_{2}}\right)=\left(x_{1}+x_{2}, v_{1}+v_{2}, \overline{w_{1}}+\overline{w_{2}}+\frac{1}{2} \overline{\left(x_{2} v_{1}-x_{1} v_{2}\right)}\right) .
$$


Então, $(G(L), \cdot)$ é um grupo algébrico nilpotente de índice 2 e a álgebra de Lie correspondente é isomorfa a $L$.

(2) A aplicação $\sigma: G \rightarrow G$ definida por $\sigma(v+x)=-(v+x):=(v+x)^{-1}, \sigma \bar{v}=\bar{v}$ é uma involução de $G$.

(3) O subconjunto $H=\{v+\bar{v} \mid v \in V\}$ é um subgrupo de $G(L)$ e temos $G(L)=$ $(X \oplus V) H$, sendo que a fatoração é exata.

(4) $X \oplus V$ é um subgrupo torcido de $G$ e a multiplicação é dada por:

$$
\left(x_{1}+v_{1}\right) *\left(x_{2}+v_{2}\right)=\left(x_{1}+x_{2}, v_{1}+v_{2}-\left(2-x_{1}-x_{2}\right)^{-1}\left(x_{2} v_{1}-x_{1} v_{2}\right)\right) .
$$

Além disso, $(X \oplus V, *)$ é um loop de Bol algébrico global.

\section{Demonstração:}

\section{2 Álgebras de Bol Críticas}

Definição 4.2.1: Seja $V$ um espaço vetorial de dimensão $n$ sobre o corpo $\mathbb{K}$ e $X \subset \operatorname{End}(V)$ um subespaço vetorial.

1) Dizemos que $X$ é irredutível quando a álgebra associativa gerada por $X$, denotada por $\langle X\rangle$, coincide com $\operatorname{End}(V)$.

2) Dizemos que $X$ é nilpotente se para cada $x \in X$ tem-se $x^{n}=0$, onde $n=\operatorname{dim} V$.

3) Dizemos que $X$ é quasenilpotente se $X$ é irredutível e para todo subespaço $X_{0} \subset X$ tem-se que $<X_{0}>$ é nilpotente.

Observação 4.2.2: 1) A existência de espaços quasenilpotentes de dimensão arbitrária foi demonstrada em [4].

2) Se $X$ é um subespaço irredutível de $\operatorname{End}(V)$, então $V$ não possui subespaços vetoriais invariantes por $X$.

3) Seja $V$ um espaço vetorial de dimensão $n$ sobre $\mathbb{K}$ e $X \subset \operatorname{End}(V)$ quasenilpotente e considere $\mathfrak{B}:=X \oplus V$. Vamos definir uma operação bilinear em $\mathfrak{B}$ da seguinte forma: para quaisquer $v, \in X, w \in V$ definimos $X X=V V=0$ e $v w=-v(w)$. Segue 
imediatamente da definição, que com esta operação, $\mathfrak{B}$ é uma álgebra de Bol.

4) Identificaremos $\phi \times V \operatorname{com} V$.

Definição 4.2.3: Seja $\mathfrak{B}$ uma álgebra de Bol. Dizemos que $\mathfrak{B}$ é crítica se são satisfeitas as seguintes condições:

1) $\mathfrak{B}$ é solúvel mas não é nilpotente.

2) Para todo ideal $\mathcal{I}$ de $\mathfrak{B}$ a álgebra de Bol $\mathfrak{B} / \mathcal{I}$ é nilpotente.

3) Qualquer subálgebra própria de $\mathfrak{B}$ é nilpotente.

O nosso próximo objetivo é provar que $\mathfrak{B}$, construída na observação acima, é uma álgebra de Bol crítica. Para isto, precisaremos de dois lemas.

Lema 4.2.4: 1) O centro da álgebra de Bol $\mathfrak{B}$ é trivial.

2) $\mathfrak{B}$ é uma álgebra de Bol solúvel que não é nilpotente.

Demonstração: 1) Seja $v+w$ um elemento do centro de $\mathfrak{B}$. Então, para qualquer $w_{1} \in V$ temos que $0=(v+w)\left(w_{1}\right)=-v\left(w_{1}\right)$, isto é, $v=0$. Da mesma forma, para todo $v_{1} \in X$ temos $0=w v_{1}=-v_{1}(w)$, isto é, $w=0$. Portanto, o centro de $\mathfrak{B}$ é trivial.

2) Primeiramente note que $\mathfrak{B} \mathfrak{B} \subset V$. De fato, dados $v+w, v_{1}+w_{1} \in \mathfrak{B}$ temos que

$$
(v+w)\left(v_{1}+w_{1}\right)=-v\left(w_{1}\right)+v_{1}(w) \in V .
$$

Logo, $\mathfrak{B}^{3}=\mathfrak{B}^{2} \mathfrak{B}^{2} \subset V V=0$, isto é, $\mathfrak{B}$ é uma álgebra de Bol solúvel de índice de solubilidade 3. Suponha que $\mathfrak{B}$ seja nilpotente de índice nilpotência $k$. Então, pelo Lema 1.4.17, $\mathfrak{B}^{k-1} \subset Z(\mathfrak{B})$, mas pelo item (1) acima, temos que $\mathfrak{B}^{k-1}=0$, o que é um absurdo.

Lema 4.2.5: 1) Os ideais de $\mathfrak{B}$ são da forma $\widetilde{X} \oplus V$, onde $\widetilde{X}$ é um subconjunto de $X$. Além disso, $V$ é o menor ideal próprio de $\mathfrak{B}$.

2) Seja $I$ um ideal de $\mathfrak{B}$. Então $\mathfrak{B} / I$ é uma álgebra de Bol nilpotente.

3) Toda subálgebra $\mathfrak{h}$ de $\mathfrak{B}$ é da forma $\widetilde{X} \oplus V_{\tilde{X}}$, onde $\widetilde{X}$ é um subconjunto de $X$ e $V_{\widetilde{X}}$ é um subespaço vetorial de $V$ invariante por $\widetilde{X}$. Além disso, toda subálgebra de $\mathfrak{B}$ é nilpotente. 
Demonstração: 1) Claramente $V$ é um ideal de $\mathfrak{B}$. Seja $\widetilde{X} \subset X$. Então dados, $v+w \in \widetilde{X} \oplus V, v_{1}+w_{1} \in \mathfrak{B}$ temos que $(v+w)\left(v_{1}+w_{1}\right)=-v\left(w_{1}\right)+v_{1}(w) \in \widetilde{X} \oplus V$, isto é, $\widetilde{X} \times V$ é um ideal de $\mathfrak{B}$ e, além disso, $V \subset \widetilde{X} \times V$. Como $W$ não possui subespaços invariantes sobre $V$, temos que $\widetilde{X} \oplus V$ são os únicos ideais próprios de $\mathfrak{B}$. Isto prova que $V$ é o menor ideal de $\mathfrak{B}$.

2) Sejam $v_{1}+w_{1}+I, v_{2}+w_{2}+I \in \mathfrak{B} / I$. Então

$$
\left(v_{1}+w_{1}+I\right)\left(v_{2}+w_{2}+I\right)=-v_{1}\left(w_{2}\right)+v_{2}\left(w_{1}\right)+I \in V+I=I,
$$

pois $V$ está contido em todo ideal próprio de $\mathfrak{B}$. Portanto $\mathfrak{B} / I$ é uma álgebra de Bol nilpotente.

3) Da mesma forma que fizemos no item (1), prova-se que todas as subálgebras de $\mathfrak{B}$ são as como dadas no enunciado. Seja $\widetilde{X}$ um subconjunto próprio de $X$. Então $<\tilde{X}>$ é nilpotente e daí segue que $\widetilde{X} \times V$ é uma álgebra de Bol nilpotente.

Teorema 4.2.6: $\quad$ Para todo $n$ natural, existem álgebras de Bol críticas de dimensão $n$.

Demonstração: A prova da existência de álgebras de Bol críticas está contida nos dois lemas acima. 


\section{Capítulo 5}

\section{Loops de Bol unipotentes de grau de nilpotência dois}

\subsection{Loops de Bol unipotentes de grau de nilpotência dois}

Nesta seção, $\mathbb{K}$ denotará um corpo algébricamente fechado.

Definição 5.1.1: Seja $\mathcal{B}$ uma álgebra de Bol. Dizemos que $\mathcal{B}$ é de Engel se $\mathcal{B}$ é nilpotente com relação à operação trilinear e satisfaz a identidade $R_{x}^{n}=0$, onde $y R_{x}=y x$.

Teorema 5.1.2: Seja $\mathcal{B}$ uma álgebra de Bol sobre um corpo de característica $\neq 2$ tal que $\operatorname{Lie}(\mathcal{B})$ é nilpotente de grau dois. Então existe um loop de Bol algébrico correspondente global se, e somente se, $\mathcal{B}$ é de Engel.

Demonstração: Seja $L$ um loop de Bol algébrico global, $\mathcal{B}$ a álgebra de Bol correspondente, $\operatorname{Lie}(\mathcal{B})=A$ a álgebra de Lie nilpotente de grau dois e $G$ o grupo algébrico correspondente. Vamos provar que $\mathcal{B}$ é de Engel. Basta provar que $R_{x}^{n}=0$ para algum $n$ natural. Se isso não fosse verdade, então existem $x, y \in \mathcal{B}$ não-nulos tais que $x y=y$.

Como $A$ é nilpotente de grau dois temos $(a, b, c)=0$ para quaisquer $a, b, c \in \mathcal{B}$ e existe a aplicação polinomial $\exp : A \rightarrow G$, onde $G$ é o grupo algébrico unipotente 
correspondente. Aqui nós usamos o fato que característica do corpo $\mathbb{K}$ não é dois. Logo $B_{0}=\mathbb{K} x \oplus \mathbb{K} y$ é uma subálgebra de $\mathcal{B}$. Se $A_{0}$ é a subálgebra de Lie, gerada por $x, y$ em $A$, então $A_{0}=\mathbb{K} x \oplus \mathbb{K} y \oplus \mathbb{K}[x, y]$. Temos $A=\mathcal{B} \oplus C$, onde $A=\mathcal{B} \oplus \mathcal{B}^{\prime}$ $\mathbf{Z}_{2}$-graduação e $C$ é uma subálgebra de $A$. Pela definição $[x, y]=x y+b_{0}, b_{0} \in C$. $\operatorname{Logo} b_{0}=[x, y]-y \in A_{0} \cap C$ e $A_{0}=\mathcal{B}_{0} \oplus C_{0}, C_{0}=A_{0} \cap C=\mathbb{K} b_{0}$. Seja $G=L H, L=$ $\exp (\mathcal{B}), H=\exp (C), G_{0}=\exp \left(A_{0}\right)$ é o subgrupo algébrico fechado que corresponde a subálgebra $A_{0}$. Como $A_{0} \cap C=\mathbb{K} b_{0}$, então $G_{0} \cap H=H_{0}=\exp \left(k b_{0}\right), \operatorname{dim} H_{0}=1$. Daqui temos que existe um loop de Bol algébrico global correspondente a álgebra de Bol $B_{0}$, contradição com Teorema 3.2.8. Logo $B$ é de Engel.

Agora seja $B$ uma álgebra de Bol de Engel tal que $A=L i e(B)$ é nilpotente de grau dois. Por indução em $\operatorname{dim} A$. Seja $G$ um grupo algébrico unipotente correspondente e $\exp : A \rightarrow G$ como acima, a aplicação exponential polinomial.

Temos $A=\mathcal{B} \oplus \mathcal{B}^{\prime}$ uma $\mathbf{Z}_{2}$-graduação tal que $A=B \oplus C, C$ é subálgebra de $A$ correspondente a álgebra de $\operatorname{Bol} \mathcal{B}$. Como $A$ é nilpotente de grau 2 e o corpo $k$ é de característica $\neq 2$ podemos definir em $A$ estrutura de um grupo unipotente correspondente com multiplicação

$$
a . b=a+b+\frac{[a, b]}{2} .
$$

Vamos denotar este grupo por $G$. Notemos que aplicação identidade $\exp : A \rightarrow$ $G=A$ neste caso é a aplicação exponencial.

Seja $G=L . H$, onde $L=B=\exp (\mathcal{B}), H=\exp (C)=C$. Claro que $L \cap H=0$.

Vamos provar que esta decomposição determina em $L$ estrutura de um loop de Bol algébrico unipotente com álgebra de Bol correspondente $B$.

Seja $\mathcal{B} \cap Z=Z_{0}$ e $Z=Z_{0} \oplus Z_{1}$, onde $Z$ é o centro de $A$. Se $C \cap Z=C_{1} \neq 0$, podemos considerar a álgebra $A / C_{1}$ e usar indução em $\operatorname{dim} A$. Por isso podemos supor que $C \cap Z=0$.

Seja $Z_{1}=\mathcal{B} \cap Z$ e $Z=Z_{1} \oplus Z_{0}$. Como $C \cap Z=0$ então para cada $z \in Z_{0}$ existe um único $\phi(z) \in B$ tal que $z-\phi(z) \in C$. Temos $[\mathcal{B}, \mathcal{B}] \subseteq \mathcal{B}^{\prime},\left(\right.$ pois $A=B \oplus B^{\prime}$ é uma $\mathbf{Z}_{2}$-graduação) logo $[\mathcal{B}, \mathcal{B}] \cap Z_{1}=0$ e podemos supor que $[\mathcal{B}, \mathcal{B}] \subseteq Z_{0}$. Logo $[a, b]=([a, b]-\phi([a, b]))+\phi([a, b])$, onde $[a, b]-\phi([a, b]) \in C, \phi([a, b]) \in \mathcal{B}$. Daqui 
definimos

$$
a b=\phi([a, b])
$$

é a multiplicação na álgebra de $\operatorname{Bol} \mathcal{B}$.

Por construção $C_{0}=\left\{z-\phi(z) \mid z \in Z_{0}\right\} \subseteq C$. Podemos supor que álgebra $A$ é gerada por $\mathcal{B}$, em outro caso substituímos $A$ por subálgebra gerada por $B$. Logo $A=\mathcal{B} \oplus Z_{0}$ e $\operatorname{dim} Z_{0}=\operatorname{dim} C$, daqui $C=C_{0}$.

Vamos achar a fórmula para multiplicação $\star$ no loop de Bol $L=B$. Seja $a, b \in B$. Temos, por(5.1):

$$
a \cdot b=a+b+\frac{[a, b]}{2}=(a \star b) \cdot c=(a \star b)+c+\frac{[a \star b, c]}{2},
$$

onde $a \star b \in L=B, c=z-\phi(z) \in C, z \in Z_{0}$. Já provamos que $[a, b],[a \star b, c] \in Z_{0}$, logo, por (5.3):

$$
a+b=a \star b-\phi(z),
$$

e disto temos

$$
\frac{[a, b]}{2}=\frac{[a \star b, c]}{2}+z=\frac{[a+b-\phi(z), z-\phi(z)]}{2}+z=-\frac{[a+b, \phi(z)]}{2}+z .
$$

Antes de continuarmos daremos uma definição e demonstraremos um lema.

Definimos $\xi: Z_{0} \rightarrow Z_{0}, \xi(z)=[\phi(z), t]$ para um elemento fixo $t \in \mathcal{B}$.

Lema 5.1.3: Seja $t \in B, z_{0} \in Z_{0}$ e $z=\sum_{i=0}^{n-1}(-1)^{i} \xi^{i}\left(z_{0}\right)$, então

$$
z+[t, \phi(z)]=z_{0}
$$

Demonstração: Temos:

$$
\phi(z) R_{t}^{i}=\phi\left(\xi^{i}(z)\right)
$$

De fato, se $i=1$, por (5.2) segue que $\phi(z) R_{t}=\phi(z) t=\phi([\phi(z), t])=\phi(\xi(z))$. Seja $\phi(z) R_{t}^{i}=\phi\left(\xi^{i}(z)\right)$, então temos 
$\phi(z) R_{t}^{i+1}=\left(\phi(z) R_{t}^{i}\right) R_{t}=\phi\left(\xi^{i}(z)\right) R_{t}=\phi\left(\xi^{i}(z)\right) t=\phi\left(\left[\phi\left(\xi^{i}(z)\right), t\right]\right)=\phi\left(\xi\left(\xi^{i}(z)\right)\right)=$ $\phi\left(\xi^{+1} i(z)\right)$.

Seja $R_{t}^{n}=0$ (este $n$ existe tal que $\mathcal{B}$ é de Engel). Logo, pelo (5.7) $\xi^{n}=0$, pois $\operatorname{ker} \phi=0$ pela definição. Agora podemos reescrever (5.6) como $z(E+\xi)=z_{0}$, onde $E(z)=z \in Z_{0}$. Logo

$(E+\xi)^{-1}=\sum_{i=0}^{n-1}(-1)^{i} \xi^{i}$ e $z(E+\xi)=z_{0}(E+\xi)^{-1}(E+\xi)=z_{0}$.

Retornemos à prova do Teorema. Lembremos que tínhamos as seguintes equações:

$$
\begin{gathered}
a+b=a \star b-\phi(z), \\
\frac{[a, b]}{2}=\frac{[a \star b, c]}{2}+z=\frac{[a+b-\phi(z), z-\phi(z)]}{2}+z=-\frac{[a+b, \phi(z)]}{2}+z .
\end{gathered}
$$

Pelo (5.9) e Lema 5.1.3 temos:

$z=\sum_{i=0}^{n-1}(-1)^{i} \xi^{i}([a, b] / 2)$, onde $\xi(x)=[\phi(x), a+b] / 2$

$\mathrm{e}$

$$
a \star b=a+b+\phi(z) .
$$

Isso mostra que $L$ com multiplicação (5.10) é um loop de Bol unipotente global.

Finalmente, enunciaremos a conjectura que motivou o teorema acima:

Conjectura: Seja $\mathcal{B}$ uma álgebra de Bol sobre um corpo de característica 0 tal que $\operatorname{Lie}(\mathcal{B})$ é nilpotente. Então o loop de Bol algébrico correspondente é global se, e somente se, $\mathcal{B}$ é de Engel. 


\section{Referências Bibliográficas}

[1] M. A. Akivis, On local algebras of a multi-dimensional three-webs. Sb. Mat. Zh. 17, No. 1 5-11. Engl. transl.: Sib. Math. J. 17, No. 1, 3-8.Zbl. 337. 53018

[2] B. T. Bouetou, Classification of solvables Lie triple systems of dimension 3. M. Sc. thesis Moscow Friendship of nations University. 1992.

[3] O. Chein, H.O. Pflugfelder, J. D. H. Smith, Quasigroups and loops: Theory and applications (Sigma series in pure mathematics), Heldermann, Berlin, 1990.

[4] J. C. G. Fernadez, A. Grishkov, M. L. R. Montoya, L. S.I. Murakami, Commutative power-associative algebras of ail-index four, to appear.

[5] A. Grishkov, G. P. Nagy, Algebraic Bol Loops, to appear

[6] E. N. Kuz'min, O. Zaidi, Solvable and semisimple bol algebras, Translated from Algebra i Logika, Vol. 32, No. 6, pp. 665Ü682, NovemberÜDecember, 1993.

[7] J. E. Humphreys, Linear Algebraic Groups, Springer-Verlag, 1998.

[8] N. Jacobson, Lie Algebras. Interscience, 1962.

[9] W. G. Lister. A structure theory of Lie triple systems. Trans. AMS. 1952

[10] A. L. Onishchik, E. B. Vinberg, Lie Groups and Lie Algebras. Springer-Verlag, 1990.

[11] H. O. Pflugfelder. Quasigroups and Loops: Introduction, Sigama Series in Pure Mathematics 7, Helderman Verlag, 1990. 
[12] L. V. Sabinin, P. O. Mikheev, Theory of Smooth Bol Loops [in Russian], UDN, Moscow, 1985.

[13] L. V. Sabinin. Analytic Quasigroups and Geometry [in Russian], UDN, Moscow 1991.

[14] L. V. Sabinin. Smooth Quasigroups and Loops (Mathematics and Its Applications), Springer; 1st edition (August 31, 1999)

[15] L. A. B. San Martin, Álgebras de Lie, Editora da Unicamp, 1999.

[16] I. R. Shafarevish, Basic algebraic geometry, Springer, 1974. 


\section{Índice Remissivo}

algebra

ampliada das derivações internas, 16

de Bol quociente, 13

de Bol solúvel, 14

de Bol, 12

de derivação, 11

envolvente maximal, 16

envolvente minimal, 16

envolvente universal, 11

algebra

ampliada, 16

de Lie, 4

algebra de Bol

crítica, 63

de Engel, 65

nilpotente, 14

algebra de Lie

envolvente, 16

nilpotente, 6

algebra de Lie

solúvel, 5

algebra tangente, 17

automorfismo

de álgebras de Lie, 5 automorfismo

$$
\text { involutivo, } 5
$$

centro

de uma álgebra de Bol, 13

companheiro, 14

derivação

de um sistema triplo de Lie, 11

elemento

unipotente, 7

grupo, 7

envolvente, 9

grupo

de multiplicações à direita, 8

grupo algébrico, 6

afim, 6

unipotente, 7

grupo de Lie Um local, 17

homomorfismo

de álgebras de Lie, 5

homomorfismo

de álgebras de Bol, 14

ideal 
de um sistema triplo de Lie, 11

de uma álgebra de Bol, 13

ideal de um STL

solúvel, 12

indice

de nilpotência, 14

indice de nilpotência, 6

isomorfismo

de álgebras de Lie, 5

isomorfismo

de álgebras de Bol, 14

loop, 7

fortemente algébrico, 9

unipotente, 8

loop

de Bol à direita, 7

loop analítico, 17

loop de Bol

algébrico, 9

loop folder, 8

multiplicação

à direita, 8

à esquerda, 8

pseudoderivação, 14

interna, 16

radical de um STL, 12

representação adjunta, 5

sistema triplo de Lie, 10 solùvel, 12

subgrupo torcido, 8

subsistema, 11 